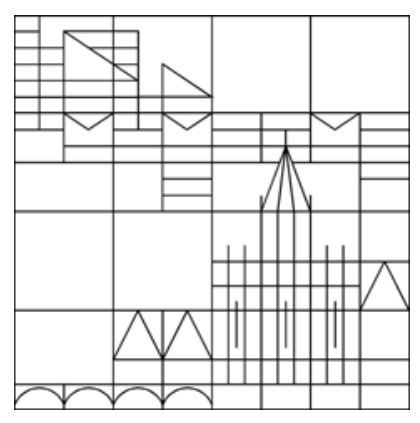

\title{
Adaptive POD basis computation for parametrized nonlinear systems using optimal snapshot location
}

\author{
Oliver Lass \\ Stefan Volkwein
}

Konstanzer Schriften in Mathematik

Nr. 304, Juni 2012

ISSN 1430-3558

Konstanzer Online-Publikations-System (KOPS)

URL: http://nbn-resolving.de/urn:nbn:de:bsz:352-191730 



\title{
ADAPTIVE POD BASIS COMPUTATION FOR PARAMETRIZED NONLINEAR SYSTEMS USING OPTIMAL SNAPSHOT LOCATION
}

\author{
Oliver Lass and Stefan Volkwein \\ Universität Konstanz \\ Fachbereich Mathematik und Statistik \\ Universitätsstraße 10 \\ D-78457 Konstanz, Germany
}

(Communicated by the associate editor name)

\begin{abstract}
The construction of reduced-order models for parametrized partial differential systems using proper orthogonal decomposition (POD) is based on the information of the so-called snapshots. These provide the spatial distribution of the nonlinear system at discrete parameter and/or time instances. In this work a strategy is used, where the POD reduced-order model is improved by choosing additional snapshot locations in an optimal way; see Kunisch and Volkwein (ESAIM: M2AN, 44:509-529, 2010). These optimal snapshot locations influences the POD basis functions and therefore the POD reduced-order model. This strategy is used to build up a POD basis on a parameter set in an adaptive way. The approach is illustrated by the construction of the POD reduced-order model for the complex-valued Helmholtz equation.
\end{abstract}

1. Introduction. Proper orthogonal decomposition (POD) is one of the most popular techniques for model reduction of nonlinear system. It was first used for signal analysis and pattern recognition, consequently in the context of dynamical systems and parametrized partial differential equations, and more recently, also for optimal control and inverse problems; see, e.g., $[8,13,16,20]$.

Let us consider the parametrized system

$$
F(y(\mu) ; \mu)=0, \quad \mu \in \mathcal{M}_{a d},
$$

in a Banach space $\mathcal{B}$, where $y(\mu)$ denotes the state associated with the parameter $\mu$ and $\mathcal{M}_{a d}$ stands for the set of admissible parameters. The parameter $\mu$ can be a coefficient (function) vector (in the case of elliptic systems) or the time variable (in the case of dynamical systems). The snapshot version of POD assumes the availability of the states $y_{j}=y\left(\mu_{j}\right) \in \mathcal{H}, 1 \leq j \leq n$, solving 1.1 for $\mu=\mu_{j}$, where $\mathcal{H}$ denotes a separable Hilbert space. Then the POD basis $\left\{\psi_{i}\right\}_{i=1}^{\ell}$ of rank $\ell \geq 1$ is

2000 Mathematics Subject Classification. Primary: 49K20, 49M15, 65N30; Secondary: 49J20, 35J15.

Key words and phrases. Model reduction, proper orthogonal decomposition, optimal snapshot locations, Helmholtz equation, optimal control, adaptive basis generation.

The author gratefully acknowledges support by the Austrian Science Fund FWF under grant no. P19588-N18. 
given by the solution to the minimization problem

$$
\begin{aligned}
& \min _{\psi_{1}, \ldots, \psi_{\ell} \in \mathcal{H}} \sum_{j=1}^{n} \alpha_{j}\left\|y_{j}-\sum_{i=1}^{\ell}\left\langle y_{j}, \psi_{i}\right\rangle_{\mathcal{H}} \psi_{i}\right\|_{\mathcal{H}}^{2} \\
& \quad \text { subject to (s.t.) }\left\langle\psi_{i}, \psi_{j}\right\rangle_{\mathcal{H}}=\delta_{i j} \text { for } 1 \leq i, j \leq \ell,
\end{aligned}
$$

where the $\alpha_{j}$ 's denote non-negative weights and $\delta_{i j}$ stands for the Kronecker symbol, i.e., $\delta_{i j}=0$ for $i \neq j$ and $\delta_{i i}=1$. Then, the solution $y(\mu)$ to 1.1 is approximated by a Galerkin ansatz of the form

$$
y^{\ell}(\mu)=\sum_{i=1}^{\ell} \mathrm{y}_{i}^{\ell}(\mu) \psi_{i}
$$

with the $\mu$-dependent coefficients $\mathrm{y}_{i}^{\ell}(\mu)$, which have to be determined. After a Galerkin projection model 1.1 is replaced by a reduced-order model of the form

$$
F^{\ell}\left(y^{\ell}(\mu) ; \mu\right)=0, \quad \mu \in \mathcal{M}_{a d} .
$$

From 1.2 it does not follow directly that the error

$$
\sum_{j=1}^{n} \alpha_{j}\left\|y\left(\mu_{j}\right)-y^{\ell}\left(\mu_{j}\right)\right\|_{\mathcal{H}}^{2}
$$

decays if the number $\ell$ of POD functions is increased. For that reason an a-priori analysis has to be carried out; see [14, 12] for certain parabolic or elliptic problems. In this paper we continue the results in [15]. We do not want to increase $\ell$, but we determine additional parameters $\left\{\tilde{\mu}_{j}\right\}_{j=1}^{n}$, the optimal snapshot locations in such a way that the new POD basis $\left\{\tilde{\psi}_{i}\right\}_{i=1}^{\ell}$ solving

$$
\begin{aligned}
& \min _{\tilde{\psi}_{1}, \ldots, \tilde{\psi}_{\ell} \in \mathcal{H}} \sum_{j=1}^{n} \alpha_{j}\left\|y_{j}-\sum_{i=1}^{\ell}\left\langle y_{j}, \tilde{\psi}_{i}\right\rangle_{\mathcal{H}} \tilde{\psi}_{i}\right\|_{\mathcal{H}}^{2}+\sum_{j=1}^{n} \tilde{\alpha}_{j}\left\|\tilde{y}_{j}-\sum_{i=1}^{\ell}\left\langle\tilde{y}_{j}, \tilde{\psi}_{i}\right\rangle_{\mathcal{H}} \tilde{\psi}_{i}\right\|_{\mathcal{H}}^{2} \\
& \text { s.t. }\left\langle\tilde{\psi}_{i}, \tilde{\psi}_{j}\right\rangle_{\mathcal{H}}=\delta_{i j} \text { for } 1 \leq i, j \leq \ell,
\end{aligned}
$$

leads to a reduced-order solution $y^{\ell}(\mu)$, so that 1.4 is as small as possible. This approach is called optimal snapshot location strategy. In 1.5 the $\tilde{\alpha}_{j}$ 's are again appropriate non-negative weights and $\tilde{y}_{j}=y\left(\tilde{\mu}_{j}\right)$ are the snapshots associated with the new snapshot locations.

Let us briefly mention some related issues of interest. In $[4,6]$ the situation of missing snapshot data is investigated and gappy POD is introduced for their reconstruction. An important alternative to POD model reduction is given by reduced-basis approximations; we refer to [19] and references given there. In [7] a reduced model is constructed for a parameter dependent family of large scale problems by an iterative procedure that adds new basis variables on the basis of a greedy algorithm. Utilizing the structure in which the parameters enter into the system this can be achieved in a computationally efficient manner. From an aposteriori analysis it follows that the error 1.4 can be bounded by a chosen tolerance on a discrete subset of $\mathcal{M}_{a d}$. In our approach, no a-priori discretization of the set $\mathcal{M}_{a d}$ is necessary. In the thesis [3] a model reduction is sought of a class for a family of models corresponding to different operating stages.

In this paper we continue our work [15], where the approach of choosing optimal snapshot location is introduced and illustrated for an linear parabolic problem. In contrast to [15] we consider a nonlinear elliptic system. More, precisely, we study 
the Helmholtz equation on a frequency band, where the frequency matches the parameter $\mu$ in the parametrized system 1.1. Let us refer to our work [9, 22, 24], where we consider impedance identification problems for the Helmholtz equation on a frequency band. In $[22,24]$ these identification problems are solved by a POD reduced-order approach. Here, we utilize the optimal snapshots locations to build up a POD basis in a successive way. Suppose that the parameter set $\mathcal{M}_{a d}$ is decomposed as follows:

$$
\mathcal{M}_{a d}=\bigcup_{i=1}^{m} \mathcal{M}_{a d}^{(i)}, \quad \mathcal{M}_{a d}^{(i)} \neq \emptyset \text { for } i=1, \ldots, m \text { and } \mathcal{M}_{a d}^{(i)} \cap \mathcal{M}_{a d}^{(j)}=\emptyset \text { for } i \neq j .
$$

Suppose that we have computed a POD basis $\left\{\psi_{i}^{(1)}\right\}_{i=1}^{\ell^{(1)}}$ of rank $\ell^{(1)} \geq 1$ utilizing solutions $\left\{y\left(\mu_{j}^{(1)}\right)\right\}_{j=1}^{n}$ to 1.1 with parameters $\mu_{1}^{(1)}, \ldots, \mu^{(n)} \in \mathcal{M}_{a d}^{(1)}$. To derive a accurate reduced-order model 1.3 for parameters $\mu \in \mathcal{M}_{a d}^{(2)}$ we modify our POD basis as follows:

- Set $\ell_{2}=\ell_{1}+\Delta \ell$ with $\Delta \ell \geq 0$

- Compute a new POD basis $\left\{\psi_{i}^{(2)}\right\}_{i=1}^{\ell^{(2)}}$ by optimal snapshot location, i.e., we add $\mathrm{n} \geq 1$ snapshots $\left\{y\left(\mu_{j}^{(2)}\right)\right\}_{j=1}^{\mathrm{n}}$ associated with optimal parameters $\mu_{1}^{(2)}, \ldots, \mu_{\mathrm{n}}^{(2)} \in \mathcal{M}_{a d}^{(2)}$, which are determined by the optimal snapshot location strategy;

- Derive the reduced-order model 1.3 utilizing the POD basis $\left\{\psi_{i}^{(2)}\right\}_{i=1}^{\ell_{2}}$.

Then, we proceed in an iterative way and generate a reduced-order model 1.3, which is reliable on the whole parameter set $\mathcal{M}_{a d}$. Let us mention that this adaptive strategy can also be used for dynamical systems, in particular for parabolic equations with a strong convection term. Here we refer the reader to the recent work [1], where the POD basis is changed for advection-diffusion problems.

The paper is organized in the following manner: In Section 2 we review the Helmholtz equation on a frequency band and introduce the finite-element model. The POD reduced-order model is derived in Section 3, where we also present an a-priori error estimate for the difference between the finite element and the POD reduced-order solution. In Section 4 we discuss the minimization problem to determine the optimal snapshot locations, which are in our application optimal frequency locations in the frequency band. Numerical experiments are carried out in Section 5. Some of the proofs are given in the Appendix.

Notation. Throughout the paper we write $\Re e(z)$ and $\Im m(z)$ for the real and imaginary part, respectively, for $z \in \mathbb{C}$. Moreover, $\bar{z}$ stands for the complex conjugate of $z$.

2. The Helmholtz equation as the large-scale model. To illustrate the proposed adaptive POD basis computation we study the complex-valued Helmholtz equation as a specific application. In an analogous manner we can proceed for other parametrized elliptic or for dynamical systems.

2.1. The Helmholtz equation. Let $\Omega \subset \mathbb{R}^{d}, d \in\{2,3\}$, be an open and bounded domain with Lipschitz-continuous boundary $\Gamma=\partial \Omega$. The domain $\Omega$ represents the interior of the car vehicle. We write $\boldsymbol{x}=\left(x_{1}, \ldots, x_{d}\right)$ for an element in $\Omega$. Recall 
that the Lebesgue space $H=L^{2}(\Omega ; \mathbb{C})$ is defined as

$$
H=\left\{\varphi: \Omega \rightarrow \mathbb{C} \mid \varphi \text { is measurable and } \int_{\Omega}|\varphi(\boldsymbol{x})|^{2} \mathrm{~d} \boldsymbol{x}<\infty\right\} .
$$

It is well-known that $H$ is a Hilbert space endowed with the inner product

$$
\langle\varphi, \bar{\psi}\rangle_{H}=\int_{\Omega} \varphi(\boldsymbol{x}) \overline{\psi(\boldsymbol{x})} \mathrm{d} \boldsymbol{x} \quad \text { for } \varphi, \psi \in H
$$

and the induced norm $\|\varphi\|_{H}=\langle\varphi, \bar{\varphi}\rangle_{H}^{1 / 2}$ for $\varphi \in H$. Note that we use standard inner product in $L^{2}(\Omega)=L^{2}(\Omega ; \mathbb{R})$ and we explicitly denote conjugates for clarity. The Hilbert space $V=H^{1}(\Omega ; \mathbb{C})$ is supplied with the inner product

$$
\langle\varphi, \bar{\psi}\rangle_{V}=\langle\varphi, \bar{\psi}\rangle_{H}+\sum_{i=1}^{d}\left\langle\frac{\partial \varphi}{\partial x_{i}}, \frac{\partial \bar{\psi}}{\partial x_{i}}\right\rangle_{H} \quad \text { for } \varphi, \psi \in V
$$

and its induced norm $\|\varphi\|_{V}=\langle\varphi, \bar{\varphi}\rangle_{V}^{1 / 2}$ for $\varphi \in V$. Recall that

$$
\begin{aligned}
\langle\psi, \bar{\varphi}\rangle_{V} & ={\overline{\langle\bar{\psi}, \varphi\rangle_{V}}=\langle\bar{\varphi}, \psi\rangle_{V}} & & \text { for all } \psi, \varphi \in V, \\
\langle\alpha(\psi+\phi), \bar{\varphi}\rangle_{V} & =\alpha\left(\langle\psi, \bar{\varphi}\rangle_{V}+\langle\phi, \bar{\varphi}\rangle_{V}\right) & & \text { for all } \psi, \phi, \varphi \in V \text { and } \alpha \in \mathbb{C}
\end{aligned}
$$

which implies that

$$
\langle\psi, \overline{\alpha(\phi+\varphi)}\rangle_{V}=\bar{\alpha}\left(\langle\psi, \bar{\varphi}\rangle_{V}+\langle\phi, \bar{\varphi}\rangle_{V}\right) \quad \text { for all } \psi, \phi, \varphi \in V \text { and } \alpha \in \mathbb{C} .
$$

For more details on Lebesgue and Sobolev spaces we refer the reader, e.g., to [5].

Next we introduce the Helmholtz equation on the frequency band

$$
\mathcal{F}=\left[f_{a}, f_{b}\right] \subset\{f \in \mathbb{R} \mid f>0\}=: \mathbb{R}^{+} .
$$

For given frequency $f \in \mathcal{F}$ the complex-valued sound pressure $p=p(f)$ solves the Helmholtz equation

$$
\begin{aligned}
-\Delta p(f)-k_{f}^{2} p(f) & =s(f) & & \text { in } \Omega, \\
\frac{\jmath}{\varrho_{\circ} \omega_{f}} \frac{\partial p}{\partial n}(f) & =0 & & \text { on } \Gamma_{\mathrm{N}} \subsetneq \Gamma=\partial \Omega, \\
\frac{\jmath}{\varrho_{\circ} \omega_{f}} \frac{\partial p}{\partial n}(f) & =A_{f} p(f) & & \text { on } \Gamma_{\mathrm{R}}=\Gamma \backslash \Gamma_{\mathrm{N}},
\end{aligned}
$$

where $s=s(f): \Omega \rightarrow \mathbb{C}$ stands for the $f$-dependent source term modelling the excitation at the point $x_{\circ} \in \Omega$ at the frequency $f$, the parameter $A_{f} \in \mathbb{C}$ denotes the $f$-dependent admittance. Notice that $Z_{f}=1 / A_{f}$ stands for the $f$-dependent impedance. Furthermore, $\jmath$ is the imaginary unit, $n$ denotes the outward normal vector and the constants in 2.6 are

$$
c=343.799 \frac{\mathrm{m}}{\mathrm{s}}, k_{f}=\frac{2 \pi f}{\mathrm{c}}, \varrho_{\circ}=1.19985 \frac{\mathrm{kg}}{\mathrm{m}^{3}}, \omega_{f}=2 \pi f=c k_{f},
$$

i.e., both the wave number $k_{f}$ and the angular frequency $\omega_{f}$ depend on $f$. In Figure 2.1 we plot the impedance and admittance values for the damping material Melamin $50 \mathrm{~mm}$ in the frequency range from 200 to $500 \mathrm{~Hz}$. Integration by parts 

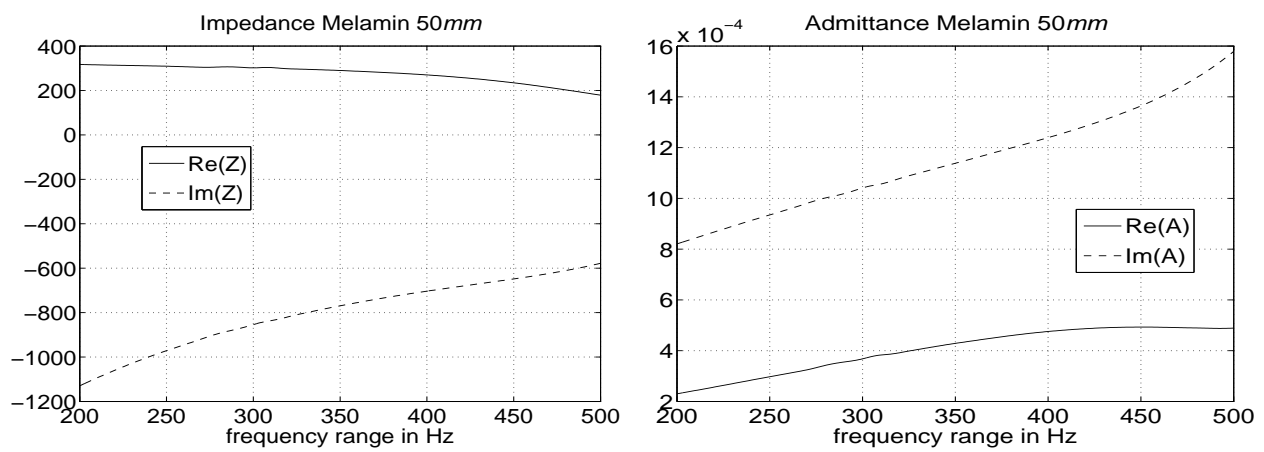

FiguRE 2.1. Impedance $Z_{f}=1 / A_{f}$ (in $\frac{P a}{\mathrm{~m} / \mathrm{s}}$ ) and admittance $A_{f}$ (in $\frac{\mathrm{m} / \mathrm{s}}{\mathrm{Pa}}$ ) values for Melamin $50 \mathrm{~mm}$ in the frequency range from 200 to $500 \mathrm{~Hz}$.

and using the boundary condition on $\Gamma_{\mathrm{R}}$ yield

$$
\begin{aligned}
\langle-\Delta p, \bar{\varphi}\rangle_{H} & =\int_{\Omega}-\Delta p \bar{\varphi} \mathrm{d} \boldsymbol{x}=-\int_{\Gamma_{\mathrm{R}}} \frac{\partial p}{\partial n} \bar{\varphi} \mathrm{d} \boldsymbol{s}+\int_{\Omega} \nabla p \cdot \nabla \bar{\varphi} \mathrm{d} \boldsymbol{x} \\
& =-\frac{1}{\jmath} \int_{\Gamma_{\mathrm{R}}} \varrho_{\circ} \omega_{f} A_{f} p \bar{\varphi} \mathrm{d} \boldsymbol{s}+\int_{\Omega} \nabla p \cdot \nabla \bar{\varphi} \mathrm{d} \boldsymbol{x} \\
& =\int_{\Omega} \nabla p \cdot \nabla \bar{\varphi} \mathrm{d} \boldsymbol{x}+\jmath \varrho_{\circ} \omega_{f} A_{f} \int_{\Gamma_{\mathrm{R}}} p \bar{\varphi} \mathrm{d} \boldsymbol{s}
\end{aligned}
$$

for every $\varphi \in V$, where we have assumed that $-\Delta p \in H$. This motivates the next definition.

Definition 2.1. For given $f>0$ the function $p(f)$ is called a weak solution to 2.6 provided $p(f) \in V$ holds and $p$ satisfies

$$
B(p, \bar{\varphi} ; f)=\int_{\Omega} s(f) \bar{\varphi} \mathrm{d} \boldsymbol{x} \quad \text { for all } \varphi \in V,
$$

where the $f$-dependent bilinear form $B(\cdot, \cdot ; f): V \times V \rightarrow \mathbb{C}$ is defined as

$$
B(p, \bar{\varphi} ; f)=\int_{\Omega} \nabla p \cdot \nabla \bar{\varphi}-k_{f}^{2} p \bar{\varphi} \mathrm{d} \boldsymbol{x}+\jmath \varrho_{\circ} \omega_{f} A_{f} \int_{\Gamma_{\mathrm{R}}} p \bar{\varphi} \mathrm{d} \boldsymbol{s}
$$

for $p, \varphi \in V$.

The existence of a weak solution to 2.6 follows from the Fredholm alternative; see, e.g., [5, pp. 640-644]. For more details we refer to [22, Theorem 2.2].

Theorem 2.2. One of the following statements hold: either

$$
\left\{\begin{array}{l}
\text { for each } s(f) \in H \text { there exists a unique weak solution } p(f) \text { to } \\
\left\{\begin{array}{rll}
-\Delta p-k_{f}^{2} p=s(f) & \text { in } \Omega, \\
\frac{j}{\varrho_{o} \omega_{f}} \frac{\partial p}{\partial n}=0 & \text { on } \Gamma_{\mathrm{N}} \\
\frac{\jmath}{\varrho_{o} \omega_{f}} \frac{\partial p}{\partial n}=A_{f} p & \text { on } \Gamma_{\mathrm{R}}
\end{array}\right.
\end{array}\right.
$$

or else

$$
\left\{\begin{array}{l}
\text { there exists a weak solution } \psi \neq 0 \text { to } \\
-\Delta \psi=k_{f}^{2} \psi \text { in } \Omega, \quad \frac{\jmath}{\varrho_{o} \omega_{f}} \frac{\partial \psi}{\partial n}=0 \text { on } \Gamma_{\mathrm{N}}, \quad \frac{\jmath}{\varrho_{o} \omega_{f}} \frac{\partial \psi}{\partial n}=A_{f} \psi \text { on } \Gamma_{\mathrm{R}} .
\end{array}\right.
$$


Remark 2.3. From the theory of compact operators and the Fredholm alternative it follows that case 2.11 appears only for a countable set of wave numbers $k_{f}$. This $f$-dependent set of countable wave numbers is denoted by $\Sigma_{f}$. Then, for all $\sigma \in \Sigma_{f}$ the problem

$$
-\Delta \psi=\sigma^{2} \psi \text { in } \Omega, \quad \frac{\jmath}{\varrho_{\circ} \omega_{f}} \frac{\partial \psi}{\partial n}=0 \text { on } \Gamma_{\mathrm{N}}, \quad \frac{\jmath}{\varrho_{\circ} \omega_{f}} \frac{\partial \psi}{\partial n}=A_{f} \psi \text { on } \Gamma_{\mathrm{R}}
$$

has a weak solution $\psi \neq 0$.

From [22, Corollaries 3.4 and 2.5] we infer the following result.

Corollary 2.4. Suppose that $k_{f} \notin \Sigma_{f}$ holds for any $f \in \mathcal{F}$. Then, 2.10 has a unique solution $p(f) \in V \cap C(\bar{\Omega} ; \mathbb{C})$ for every source term $s(f) \in H$ satisfying

$$
\|p(f)\|_{V}+\|p(f)\|_{C(\bar{\Omega} ; \mathbb{C})} \leq K_{f},
$$

where the constant $K_{f}>0$ depends on $f$. In particular, $K_{f}$ blows up if $k_{f}$ approaches an element in $\Sigma_{f}$.

Let us define the $f$-dependent bounded linear operator $\mathcal{A}_{f}: V \rightarrow V^{\prime}$ by

$$
\mathcal{A}_{f} \varphi=B(\varphi, \cdot ; f) \text { for } \varphi \in V .
$$

By Corollary 2.4 the operator $\mathcal{A}_{f}$ is continuously invertible for any $f \in \mathcal{F}$. Moreover, let the frequency dependent functional $\mathcal{S}_{f} \in V^{\prime}$ be introduced by

$$
\left\langle\mathcal{S}_{f}, \bar{\varphi}\right\rangle_{V^{\prime}, V}=\langle s(f), \bar{\varphi}\rangle_{H} \quad \text { for } \varphi \in V .
$$

Then we can write 2.6 as the following operator equation

$$
\mathcal{A}_{f} p(f)=\mathcal{S}(f) \text { in } V^{\prime} \text { for almost all } f \in \mathcal{F} \text {. }
$$

Notice that the dual operator $\mathcal{A}_{f}^{\star}: V \rightarrow V^{\prime}$ of $\mathcal{A}_{f}$ satisfying

$$
\left.\left\langle\mathcal{A}_{f}^{\star} \varphi, \bar{\psi}\right\rangle_{V^{\prime}, V}=\overline{\langle\mathcal{A}}_{f} \psi, \bar{\varphi}\right\rangle_{V^{\prime}, V} \quad \text { for all } \varphi, \psi \in V
$$

is given by

$$
\begin{aligned}
\left\langle\mathcal{A}_{f}^{\star} \varphi, \bar{\psi}\right\rangle_{V^{\prime}, V} & ={\overline{\langle\mathcal{A} f} \psi, \bar{\varphi}\rangle_{V^{\prime}, V}} \\
& =\int_{\Omega} \nabla \varphi \cdot \nabla \bar{\psi}-k_{f}^{2} \varphi \bar{\psi} \mathrm{d} \boldsymbol{x}-\jmath \varrho_{\circ} \omega_{f} \bar{A}_{f} \int_{\Gamma_{\mathrm{R}}} \varphi \bar{\psi} \mathrm{d} \boldsymbol{s}
\end{aligned}
$$

for $\varphi, \psi \in V$. Next we consider the mapping $\mathcal{F} \ni f \mapsto p(f) \in V$. For that purpose we make use of the following assumption.

Assumption 1. For given $A \in C(\mathcal{F} ; \mathbb{C})$ and $s \in C(\mathcal{F} ; H)$ there exists a $\delta=$ $\delta(A, s)>0$ such that $\inf _{f \in \mathcal{F}} \inf _{\sigma \in \Sigma_{f}}\left|k_{f}-\sigma\right| \geq \delta$ holds.

Remark 2.5. Let $A \in C(\mathcal{F} ; \mathbb{C})$ and $s \in C(\mathcal{F} ; H)$. As before we write $A_{f} \in \mathbb{C}$ for the function $f \mapsto A_{f}$. With Assumption 1 holding 2.6 is uniquely solvable for any $f \in \mathcal{F}$ and the constant $K$ in Corollary 2.4 can be chosen to be independent of $f$. Hence, there exists a constant $K>0$ satisfying

$$
\max _{f \in \mathcal{F}}\left(\|p(f)\|_{V}+\|p(f)\|_{C(\bar{\Omega} ; \mathbb{C})}\right) \leq K,
$$

where $K$ is independent of $f$. 
To determine optimal snapshot location we have to linearize the Helmholtz equation with respect to the frequency. For this purpose we suppose that $s \in H^{1}(\mathcal{F} ; H)$ and $A \in H^{1}(\mathcal{F} ; \mathbb{C})$. Then, $s \in C(\mathcal{F} ; H)$ and $A \in C(\mathcal{F} ; \mathbb{C})$ holds. Notice that $\dot{\mathcal{A}}_{f}: V \rightarrow V^{\prime}$ and $\dot{\mathcal{S}}_{f} \in V^{\prime}$ are given by

$$
\begin{aligned}
& \dot{\mathcal{A}}_{f} \varphi=\jmath \varrho_{\circ}\left(\omega_{f} \dot{A}_{f}+\dot{\omega}_{f} A_{f}\right)\langle\varphi, \cdot\rangle_{L^{2}\left(\Gamma_{\mathrm{R}} ; \mathbb{C}\right)}-2 k_{f} \dot{k}_{f}\langle\varphi, \cdot\rangle_{H} \in V^{\prime}, \\
& \left\langle\dot{\mathcal{S}}_{f}, \bar{\varphi}\right\rangle_{V^{\prime}, V}=\langle\dot{s}(f), \bar{\varphi}\rangle_{H}
\end{aligned}
$$

for $\varphi \in V$. Clearly, $\dot{\mathcal{A}}_{f}$ and $\dot{\mathcal{S}}_{f}$ are bounded and linear operators for all $f \in \mathcal{F}$. We differentiate 2.13 with respect to $f$. By $\dot{p}(f)$ we denote the derivative of $p$ with respect to $f$. It follows for almost all $f \in \mathcal{F}$

$$
\mathcal{A}_{f} \dot{p}(f)=\dot{\mathcal{S}}_{f}-\dot{\mathcal{A}}_{f} p(f) \text { in } V^{\prime} \text { for almost all } f \in \mathcal{F} \text {. }
$$

The proof of the next result is discussed in [22, Corollary 2.8].

Corollary 2.6. Let Assumption 1 be satisfied. Suppose that $A \in H^{1}(\mathcal{F} ; \mathbb{C})$ and $q \in H^{1}(\mathcal{F} ; H)$. Then, $p \in H^{1}(\mathcal{F} ; C(\bar{\Omega} ; \mathbb{C}) \cap V)$. In particular, there exists a constant $K>0$ (independent of $f$ ) such that

$$
\max _{f \in \mathcal{F}}\left(\|\dot{p}(f)\|_{V}+\|\dot{p}(f)\|_{C(\bar{\Omega} ; \mathbb{C})}\right) \leq K .
$$

2.2. The finite element model. Suppose that $\varphi_{1}, \ldots, \varphi_{N} \in H^{1}(\Omega ; \mathbb{R})$ denote linearly independent finite element functions. We set

$$
V^{N}=\left\{z^{N}=\sum_{i=1}^{N} \mathrm{z}_{i}^{N} \varphi_{i} \in V \mid \mathrm{z}_{i}^{N} \in \mathbb{C} \text { for } i=1, \ldots, N\right\} \subset V .
$$

A finite element solution to 2.8 is given as follows: $p^{N} \in V^{N}$ satisfies

$$
B\left(p^{N}, \varphi^{N} ; f\right)=\left\langle\mathcal{S}_{f}, \varphi^{N}\right\rangle_{V^{\prime}, V} \text { for all } \varphi^{N} \in V^{N} .
$$

Inserting the expression $p^{N}=\sum_{j=1}^{N} \mathrm{p}_{j} \varphi_{j}$ into 2.16 and choosing $\varphi^{N}=\varphi_{i}$ for $1 \leq i \leq N$ we arrive at

$$
\sum_{j=1}^{N} B\left(\varphi_{j}, \varphi_{i} ; f\right) \mathrm{p}_{j}=\left\langle\mathcal{S}_{f}, \varphi_{i}\right\rangle_{V^{\prime}, V} \quad \text { for all } 1 \leq i \leq N .
$$

Thus, 2.16 can be represented by the linear system

$$
\mathrm{B}_{f} \mathrm{p}=\mathrm{s}_{f} \in \mathbb{C}^{N}
$$

where we set the matrix $\mathrm{B}_{f}$ as

$$
\mathrm{B}_{f}=\left(\left(B\left(\varphi_{j}, \varphi_{i} ; f\right)\right)\right)_{1 \leq i, j \leq N}=\left(\left(\left\langle\mathcal{A}_{f} \varphi_{j}, \varphi_{i}\right\rangle_{V^{\prime}, V}\right)\right)_{1 \leq i, j \leq N}
$$

and the two vectors $\mathrm{p}, \mathrm{s}_{f}$ by

$$
\mathrm{p}=\left(\mathrm{p}_{i}\right)_{1 \leq i \leq N}, \quad \mathrm{~s}_{f}=\left(\left\langle\mathcal{S}_{f}, \varphi_{i}\right\rangle_{V^{\prime}, V}\right)_{1 \leq i \leq N}=\left(\left\langle s(f), \varphi_{i}\right\rangle_{H}\right)_{1 \leq i \leq N} .
$$

Introducing the $f$-independent matrices

$$
\begin{aligned}
& \mathrm{S}=\left(\left(\int_{\Omega} \nabla \varphi_{j} \cdot \nabla \varphi_{i} \mathrm{~d} \boldsymbol{x}\right)\right)_{1 \leq i, j \leq N}, \quad \mathrm{M}=\left(\left(\int_{\Omega} \varphi_{j} \varphi_{i} \mathrm{~d} \boldsymbol{x}\right)\right)_{1 \leq i, j \leq N}, \\
& \mathrm{Q}=\left(\left(\jmath \varrho_{\Gamma_{\mathrm{R}}} \varphi_{j} \varphi_{i} \mathrm{~d} \boldsymbol{s}\right)\right)_{1 \leq i, j \leq N}
\end{aligned}
$$


the matrix $\mathrm{B}_{f}$ has the form $\mathrm{B}_{f}=\mathrm{S}-k_{f}^{2} \mathrm{M}+\omega_{f} A_{f} \mathrm{Q}$. If Assumption 1 holds true, the matrix $\mathrm{B}_{f}$ is regular for any $f \in \mathcal{F}$ by Remark 2.5. Thus, 2.17 is uniquely solvable. Consequently, 2.16 has a unique finite element solution $p^{N}$ for any $f \in \mathcal{F}$.

3. POD Galerkin scheme for the Helmholtz equation. In this section we recall briefly the POD method and explain the reduced-order model for the Helmholtz equation.

3.1. The POD method. Let $\mathcal{F}=\left[f_{a}, f_{b}\right] \subset \mathbb{R}^{+}$be a given frequency band satisfying Assumption 1. Then, there exists a unique finite element solution $p^{N}(f) \in V^{N}$ to 2.16 and $\mathrm{p}(f)=\left(\mathrm{p}_{i}(f)\right)_{1 \leq i \leq N}$ to 2.17. Suppose that

$$
f_{a} \leq f_{1}<f_{2}<\ldots<f_{n} \leq f_{b}
$$

is a frequency grid in $\mathcal{F}$. We set $p_{f_{j}}^{N}=p^{N}\left(f_{j}\right)$ and $\mathrm{p}_{f_{j}}=\mathrm{p}\left(f_{j}\right)$ for $1 \leq j \leq n$. Let us define the subspace $\mathcal{V}=\operatorname{span}\left\{\mathrm{p}_{f_{1}}, \ldots, \mathrm{p}_{f_{n}}\right\} \subset \mathbb{R}^{N}$ with $d^{N}=\operatorname{dim} \mathcal{V} \leq \min (n, N)$.

We define the following weighted inner product in $\mathbb{C}^{N}$ :

$$
\langle\mathrm{u}, \overline{\mathrm{v}}\rangle_{\mathrm{W}}=\mathrm{u}^{\top} \mathrm{W} \overline{\mathrm{v}}=\sum_{i=1}^{N} \sum_{j=1}^{N} \mathrm{u}_{i} \mathrm{~W}_{i j} \overline{\mathrm{v}}_{j}, \quad \mathrm{u}=\left(\mathrm{u}_{i}\right)_{1 \leq i \leq N}, \mathrm{v}=\left(\mathrm{v}_{i}\right)_{1 \leq i \leq N} \in \mathbb{C}^{N}
$$

with $\mathrm{W}=\mathrm{S}+\mathrm{M} \in \mathbb{R}^{N \times N}$. Throughout, the symbol "T" stands for transpose of a vector or matrix. Moreover, we set $|\mathrm{u}|_{\mathrm{W}}=\langle\mathrm{u}, \overline{\mathrm{u}}\rangle_{\mathrm{W}}^{1 / 2}$. Notice that $\mathrm{W}^{\top}=\mathrm{W}$ holds and $\mathrm{W}=\left(\left(\left\langle\varphi_{j}, \varphi_{i}\right\rangle_{V}\right)\right)_{1 \leq i, j \leq N}$.

Remark 3.1. For $u^{N}=\sum_{i=1}^{N} \mathrm{u}_{i} \varphi_{i}, v^{N}=\sum_{j=1}^{N} \mathrm{v}_{j} \varphi_{j} \in V^{N}$ we have

$$
\left\langle u^{N}, \bar{v}^{N}\right\rangle_{V}=\sum_{i=1}^{N} \sum_{j=1}^{N} \mathrm{u}_{i} \overline{\mathrm{v}}_{j}\left\langle\varphi_{i}, \varphi_{j}\right\rangle_{V}=\sum_{i=1}^{N} \sum_{j=1}^{N} \mathrm{u}_{i} \mathrm{~W}_{j i} \overline{\mathrm{v}}_{j}=\sum_{i=1}^{N} \sum_{j=1}^{N} \mathrm{u}_{i} \mathrm{~W}_{i j} \overline{\mathrm{v}}_{j}=\langle\mathrm{u}, \overline{\mathrm{v}}\rangle_{\mathrm{W}}
$$

for vectors $\mathrm{u}=\left(\mathrm{u}_{i}\right)_{1 \leq i \leq N}$ and $\mathrm{v}=\left(\mathrm{v}_{i}\right)_{1 \leq i \leq N}$ in $\mathbb{C}^{N}$. Moreover, $\left\|u^{N}\right\|_{V}=|\mathrm{u}|_{\mathrm{W}}$ is satisfied. Thus, the $V$-topology in the finite element space corresponds to the $\mathrm{W}$-topology in the coefficient space $\mathbb{C}^{N}$. For $\mathrm{W}=\mathrm{M}$ the $H$-topology in $V^{N}$ is equivalent to the $\mathrm{W}$-topology in $\mathbb{C}^{N}$.

For an arbitrary $\ell \in\left\{1, \ldots, d^{N}\right\}$ we consider the minimization problem

$$
\begin{gathered}
\underset{\mathrm{u}_{1}, \ldots, \mathrm{u}_{\ell} \in \mathbb{C}^{N}}{ } \sum_{j=1}^{n} \alpha_{j}\left|\mathrm{p}_{f_{j}}-\sum_{i=1}^{\ell}\left\langle\mathrm{p}_{f_{j}}, \overline{\mathrm{u}}_{i}\right\rangle_{\mathrm{W}} \mathrm{u}_{i}\right|_{\mathrm{W}}^{2} \\
\text { s.t. }\left\langle\mathrm{u}_{i}, \overline{\mathrm{u}}_{j}\right\rangle_{\mathrm{W}}=\delta_{i j} \text { for } 1 \leq i, j \leq \ell,
\end{gathered}
$$

where $\left\{\alpha_{j}\right\}_{j=1}^{n}$ are nonnegative weights, $\delta_{i j}$ stands for the Kronecker symbol, i.e., $\delta_{i i}=1$ and $\delta_{i j}=0$ for $j \neq i$. A solution to $\mathbf{P}^{\ell}$ is called a POD basis of rank $\ell$.

The solution to $\mathbf{P}^{\ell}$ is given by singular value decomposition. Let us define the linear and bounded operator $\mathcal{R}: \mathbb{C}^{N} \rightarrow \mathbb{C}^{N}$ by

$$
\mathcal{R} \mathrm{u}=\sum_{j=1}^{n} \alpha_{j}\left\langle\mathrm{u}, \overline{\mathrm{p}}_{f_{j}}\right\rangle_{\mathrm{W}} \mathrm{p}_{f_{j}}=\sum_{j=1}^{n} \alpha_{j}\left\langle\overline{\mathrm{p}}_{f_{j}}, \mathrm{u}\right\rangle_{\mathrm{W}} \mathrm{p}_{f_{j}} \quad \text { for } \mathrm{u} \in \mathbb{C}^{N}
$$


Moreover, $\mathcal{R}$ is symmetric and non-negative. In fact, we obtain

$$
\begin{aligned}
\langle\mathcal{R} \mathrm{u}, \overline{\mathrm{v}}\rangle_{\mathrm{W}} & =\sum_{j=1}^{n} \alpha_{j}\left\langle\mathrm{u}, \overline{\mathrm{p}}_{f_{j}}\right\rangle_{\mathrm{W}}\left\langle\mathrm{p}_{f_{j}}, \overline{\mathrm{v}}\right\rangle_{\mathrm{W}}=\left\langle\mathrm{u}, \sum_{j=1}^{n} \alpha_{j}\left\langle\mathrm{p}_{f_{j}}, \overline{\mathrm{v}}\right\rangle_{\mathrm{W}} \overline{\mathrm{p}}_{f_{j}}\right\rangle_{\mathrm{W}} \\
& =\left\langle\mathrm{u}, \sum_{j=1}^{n} \overline{\alpha_{j}\left\langle\mathrm{v}, \overline{\mathrm{p}}_{f_{j}}\right\rangle_{\mathrm{W}} \mathrm{p}_{f_{j}}}\right\rangle_{\mathrm{W}}=\langle\mathrm{u}, \overline{\mathcal{R} \mathrm{v}}\rangle_{\mathrm{W}} \quad \text { for all } \mathrm{u}, \mathrm{v} \in \mathbb{C}^{N}
\end{aligned}
$$

and

$$
\langle\mathcal{R} \mathrm{u}, \overline{\mathrm{u}}\rangle_{\mathrm{W}}=\sum_{j=1}^{n} \alpha_{j}\left\langle\mathrm{u}, \overline{\mathrm{p}}_{f_{j}}\right\rangle_{\mathrm{W}}\left\langle\overline{\mathrm{u}}, \mathrm{p}_{f_{j}}\right\rangle_{\mathrm{W}}=\sum_{j=1}^{n} \alpha_{j}\left|\left\langle\mathrm{u}, \overline{\mathrm{p}}_{f_{j}}\right\rangle_{\mathrm{W}}\right|^{2} \geq 0 \quad \text { for all } \mathrm{u} \in \mathbb{C}^{N} .
$$

Is is proved in $[10,23]$, for instance, that the solution to $\left(\mathbf{P}^{\ell}\right)$ is given by the symmetric eigenvalue problem

$$
\mathcal{R} \mathrm{u}_{i}=\lambda_{i} \mathrm{u}_{i} \quad \text { for } i=1, \ldots, \ell,
$$

where $\lambda_{1} \geq \lambda_{2} \geq \ldots \geq \lambda_{\ell}>0$ denote the largest eigenvalues of the operator $\mathcal{R}$.

3.2. The reduced-order model for the Helmholtz equation. Suppose that we have computed a POD basis $\left\{u_{i}\right\}_{i=1}^{\ell}$ of rank $\ell$. Then, we define the matrix

$$
\Psi=\left(\mathrm{u}_{1}|\ldots| \mathrm{u}_{\ell}\right) \in \mathbb{C}^{N \times \ell}
$$

which has rank $\ell$. We can associate with each columns of $\Psi$ a finite element function as follows:

$$
\psi_{j}: \Omega \rightarrow \mathbb{C}, \quad \psi_{j}(\boldsymbol{x})=\sum_{i=1}^{N} \Psi_{i j} \varphi_{i}(\boldsymbol{x}) \quad \text { for } 1 \leq j \leq \ell .
$$

We conclude from $\left\langle\mathrm{u}_{i}, \overline{\mathrm{u}}_{j}\right\rangle_{\mathrm{W}}=\delta_{i j}$ that

$$
\left\langle\psi_{i}, \bar{\psi}_{j}\right\rangle_{V}=\left\langle\mathrm{u}_{i}, \overline{\mathrm{u}}_{j}\right\rangle_{\mathrm{W}}=\delta_{i j} \quad \text { for } 1 \leq i, j \leq \ell .
$$

Thus, the functions $\left\{\psi_{i}\right\}_{i=1}^{\ell}$ are linearly independent in $V$. We introduce the $\ell$ dimensional subspace $V^{\ell}=\operatorname{span}\left\{\psi_{1}, \ldots, \psi_{\ell}\right\}$ of $V^{N}$ and consider - instead of 2.16 - the following POD Galerkin scheme for 2.6: $p^{\ell} \in V^{\ell}$ solves

$$
B\left(p^{\ell}, \bar{\psi} ; f\right)=\int_{\Omega} s(f) \bar{\psi} \mathrm{d} \boldsymbol{x} \quad \text { for all } \psi \in V^{\ell} .
$$

From $p^{\ell} \in V^{\ell}$ we infer the representation $p^{\ell}=\sum_{j=1}^{\ell} \eta_{j} \psi_{j}$. Thus, 3.2 is equivalent to the linear equation system

$$
\sum_{j=1}^{\ell} B\left(\psi_{j}, \bar{\psi}_{i} ; f\right) \eta_{j}=\left\langle\mathcal{S}_{f}, \bar{\psi}_{i}\right\rangle_{V^{\prime}, V} \quad \text { for } 1 \leq i \leq \ell .
$$

Defining the matrix

$$
\mathrm{B}_{f}^{\ell}=\left(\left(B\left(\psi_{j}, \bar{\psi}_{i} ; f\right)\right)\right)_{1 \leq i, j \leq \ell}=\left(\left(\left\langle\mathcal{A}_{f} \psi_{j}, \bar{\psi}_{i}\right\rangle_{V^{\prime}, V}\right)\right)_{1 \leq i, j \leq \ell}
$$

and the two vectors

$$
\eta=\left(\eta_{i}\right)_{1 \leq i \leq \ell}, \quad \mathrm{s}_{f}^{\ell}=\left(\left\langle\mathcal{S}_{f}, \bar{\psi}_{i}\right\rangle_{V^{\prime}, V}\right)_{1 \leq i \leq \ell}=\left(\left\langle s(f), \bar{\psi}_{i}\right\rangle_{H}\right)_{1 \leq i \leq \ell}
$$

we can write 3.3 in the form

$$
\mathrm{B}_{f}^{\ell} \eta=\mathrm{s}_{f}^{\ell} \in \mathbb{C}^{\ell}
$$


If $\ell \ll N$ holds, 3.4 is a reduced-order or low-dimensional model for 2.17. Notice that $\mathrm{B}_{f}^{\ell}$ and $\mathrm{s}_{f}^{\ell}$ can be computed from the finite element representation by utilizing the above defined matrix $\Psi$ as a projection matrix:

$$
\mathrm{B}_{f}^{\ell}=\bar{\Psi}^{\top} \mathrm{B}_{f} \Psi=\bar{\Psi}^{\top}\left(\mathrm{S}-k_{f}^{2} \mathrm{M}+\omega_{f} A_{f} \mathrm{Q}\right) \Psi, \quad \mathrm{s}_{f}^{\ell}=\bar{\Psi}^{\top} \mathrm{s}_{f} .
$$

If Assumption 1 is satisfied, the matrix $\mathrm{B}_{f}$ is regular for all $f \in \mathcal{F}$. Then, $\mathrm{B}_{f}^{\ell}$ is also regular for all $f \in \mathcal{F}$. Thus, 3.4 possesses a unique solution $\mathrm{p}^{\ell}=\left(\mathrm{p}_{i}^{\ell}\right)_{1 \leq i \leq \ell}$. Moreover, $p^{\ell}=\sum_{i=1}^{\ell} \mathrm{p}_{i}^{\ell} \psi_{i}$ is the unique solution to 3.2 .

The error between a solutions to 2.16 and to 3.2 can be bounded by an a-priori estimates. For that reason we need the next assumption.

Assumption 2. For $f \in \mathcal{F}$ let $\Sigma_{f}^{\ell}$ denote the set of singular values $\sigma$ so that

$$
\mathrm{B}_{f}^{\ell} \mathrm{u}=\sigma^{2} \mathrm{Mu}
$$

has a solution $\mathrm{u} \in \mathbb{C}^{N} \backslash\{0\}$. Then there exists a $\delta>0$ independent of $\ell$ and $N$ such that $\inf _{f \in \mathcal{F}} \inf _{\sigma \in \Sigma_{f}^{\ell}}\left|k_{f}-\sigma\right| \geq \delta$ hold.

The following results follows from [22, Proposition 4.1].

Proposition 3.2. With Assumption 2 holding there is a constant $C>0$ independent of $\ell$ and $N$ satisfying

$$
\sum_{j=1}^{n} \alpha_{j}\left\|p^{N}\left(f_{j}\right)-p^{\ell}\left(f_{j}\right)\right\|_{V}^{2} \leq C \sum_{i=\ell+1}^{d^{N}} \lambda_{i} .
$$

4. Optimal snapshot location. Suppose that Assumption 1 holds. The POD basis $\left\{\mathrm{u}_{i}\right\}_{i=1}^{\ell}$ of rank $\ell$ is determined from knowledge of the solution $\left\{\mathrm{p}_{f_{j}}\right\}_{j=1}^{n}$ at discrete frequencies $f_{j} \in \mathcal{F}$. We distinguish here between a predefined set chosen on a uniform distributed frequency band and additional frequencies which will be determined in an optimal way [15].

4.1. The optimal control problem. Let $n$ be the number of fixed chosen frequencies $\left\{f_{j}\right\}_{j=1}^{n}$ with associated solutions $\mathrm{p}_{f_{j}}=\mathrm{p}\left(f_{j}\right) \in \mathbb{C}^{N}$ to 2.17. The snapshots at new frequencies $\mathrm{f}_{k} \in \mathcal{F}, 1 \leq k \leq \mathrm{n}$, are denoted as $\mathrm{p}_{\mathrm{f}_{k}}=\mathrm{p}\left(\mathrm{f}_{k}\right)$. We set

$$
\mathcal{V}=\operatorname{span}\left\{\mathrm{p}_{f_{1}}, \ldots, \mathrm{p}_{f_{n}}, \mathrm{p}_{\mathrm{f}_{1}}, \ldots, \mathrm{p}_{\mathrm{f}_{n}}\right\} \subset \mathbb{C}^{N}
$$

with $d^{N}=\operatorname{dim} \mathcal{V} \leq \min (n+\mathrm{n}, N)$. Let $\ell \in\left\{1, \ldots, d^{N}\right\}$ be the number of chosen POD basis functions. Throughout we denote by $f$ the vector $\left(f_{1}, \ldots, f_{n}\right) \in \mathbb{R}^{n}-$ for simplicity - and define the bounded linear symmetric operator

$$
\mathcal{R}(\mathrm{f}) \mathrm{u}=\sum_{j=1}^{n}\left\langle\mathrm{u}, \overline{\mathrm{p}}_{f_{j}}\right\rangle_{\mathrm{W}} \mathrm{p}_{f_{j}}+\sum_{k=1}^{\mathrm{n}}\left\langle\mathrm{u}, \overline{\mathrm{p}}_{\mathrm{f}_{k}}\right\rangle_{\mathrm{W}} \mathrm{p}_{\mathrm{f}_{k}} \quad \text { for } \mathrm{u} \in \mathbb{C}^{N} .
$$

As described in Section 3.1, the POD basis of rank $\ell$ is given by the solution to the eigenvalue problem

$$
\mathcal{R}(\mathrm{f}) \mathrm{u}_{i}=\lambda_{i} \mathrm{u}_{i}, \quad 1 \leq i \leq \ell,
$$

where the eigenvalues are ordered and for the simplicity of the presentation are supposed to be simple:

$$
\lambda_{1}>\lambda_{2}>\ldots>\lambda_{\ell}>0 .
$$

Thus it follows that $\left\langle\mathrm{u}_{i}, \overline{\mathrm{u}}_{j}\right\rangle_{\mathrm{W}}=0$ for $1 \leq i \leq \ell$ with $i \neq j$ and we assume that

$$
\left|\mathrm{u}_{i}\right|_{\mathrm{W}}=1 \quad \text { for } 1 \leq i \leq \ell \text {. }
$$


Next we formulate the optimization problem. For that purpose we define the Hilbert spaces

$$
X=H^{1}\left(\mathcal{F} ; \mathbb{C}^{\ell}\right) \times \mathbb{R}^{\mathrm{n}} \times\left(\mathbb{C}^{N}\right)^{\ell} \times \mathbb{R}^{\ell}, \quad y=L^{2}(\mathcal{F} ; \mathbb{C}) \times\left(\mathbb{C}^{N}\right)^{\ell} \times \mathbb{R}^{\ell}
$$

endowed with their natural product topology and with

$$
\left(\mathbb{C}^{N}\right)^{\ell}=\underbrace{\mathbb{C}^{N} \times \ldots \times \mathbb{C}^{N}}_{\ell \text {-times }} .
$$

Throughout we denote by $x=(\eta, \mathrm{f}, \mathrm{u}, \lambda)$ an element in $\mathcal{X}$, where $\eta=\left(\eta_{i}\right)_{1 \leq i \leq \ell}$, $\mathrm{f}=\left(\mathrm{f}_{i}\right)_{1 \leq i \leq \mathrm{n}}, \mathrm{u}=\left(\mathrm{u}_{i}\right)_{1 \leq i \leq \ell}$ and $\lambda=\left(\lambda_{i}\right)_{1 \leq i \leq \ell}$ hold. Furthermore, $\xi=(\mathrm{q}, \mu, \phi)$ stands for an element in $\bar{y}$ with $\mathrm{q}=\left(\mathrm{q}_{i}\right)_{1 \leq i \leq \ell}, \mu=\left(\mu_{i}\right)_{1 \leq i \leq \ell}$ and $\phi=\left(\phi_{i}\right)_{1 \leq i \leq \ell}$.

To quantify the difference between the solution $p^{N}(f)=\sum_{i=1}^{\ell} \mathrm{p}_{i}(f) \varphi_{i}$ to 2.16 and the solution $p^{\ell}(f)=\sum_{i=1}^{\ell} \eta_{i}(f) \psi_{i}$ to 3.2 we introduce the cost functional $J: X \rightarrow \mathbb{R}$ by

$$
J(x)=\frac{\beta}{2} \int_{\mathcal{F}}\left\|p^{N}(f)-p^{\ell}(f)\right\|_{V}^{2} \mathrm{~d} f
$$

for $\mathrm{p}(f)=\left(\mathrm{p}_{i}(f)\right)_{1 \leq i \leq N}$ and $x=(\eta, \mathrm{f}, \psi, \lambda) \in \mathcal{X}$, where $\beta>0$ is a scaling parameter. It follows from the a-priori estimate of Proposition 3.2 that the cost $J$ decreases provided we increase the number $\ell$ of POD basis functions in the Galerkin scheme. For the optimal snapshot location we fix the number $\ell$ and add additional snapshots $\mathrm{p}_{\mathrm{f}_{k}}, 1 \leq k \leq \mathrm{n}$, in an optimal way so that the cost $J$ is minimized. From

$$
\psi_{j}(\boldsymbol{x})=\sum_{i=1}^{N} \Psi_{i j} \varphi_{i}(\boldsymbol{x})=\sum_{i=1}^{N}\left(\mathrm{u}_{j}\right)_{i} \varphi_{i} \quad \text { and } \quad p^{\ell}(f)=\sum_{j=1}^{\ell} \eta_{j}(f) \psi_{j}(\boldsymbol{x})
$$

we infer that

$$
\begin{aligned}
\left(p^{N}(f)-p^{\ell}(f)\right)(\boldsymbol{x}) & =\sum_{i=1}^{N}\left(\mathrm{p}(f)-\sum_{j=1}^{\ell} \eta_{j}(f) \mathrm{u}_{j}\right)_{i} \varphi_{i}(\boldsymbol{x}) \\
& =\sum_{i=1}^{N}(\mathrm{p}(f)-\Psi \eta(f))_{i} \varphi_{i}(\boldsymbol{x})=\sum_{i=1}^{N}\left(\mathrm{p}(f)-\mathrm{p}^{\ell}(f)\right)_{i} \varphi_{i}(\boldsymbol{x}),
\end{aligned}
$$

where $\left(\mathrm{u}_{j}\right)_{i}$ denotes the $i$-th component of the vector $\mathrm{u}_{j}$ and we set $\mathrm{p}^{\ell}(f)=\Psi \eta(f)$. Thus,

$$
J(x)=\frac{\beta}{2} \int_{\mathcal{F}}\left\|p^{N}(f)-p^{\ell}(f)\right\|_{V}^{2} \mathrm{~d} f=\frac{\beta}{2} \int_{\mathcal{F}}\left|\mathrm{p}(f)-\mathrm{p}^{\ell}(f)\right|_{\mathrm{W}}^{2} \mathrm{~d} f
$$

holds. We write the equality constraints as an operator equation. Therefore, we define the nonlinear mapping $e=\left(e_{1}, e_{2}, e_{3}\right): x \rightarrow y$ by

$$
\begin{aligned}
& e_{1}(x)=\mathrm{B}_{f}^{\ell} \mathrm{p}^{\ell}(f)-\mathrm{s}_{f}^{\ell} \in H^{1}\left(\mathcal{F} ; \mathbb{C}^{\ell}\right), \quad e_{2}(x)=\left(\begin{array}{c}
\left(\mathcal{R}(\mathrm{f})-\lambda_{1}\right) \mathrm{u}_{1} \\
\vdots \\
\left(\mathcal{R}(\mathrm{f})-\lambda_{\ell}\right) \mathrm{u}_{\ell}
\end{array}\right) \in\left(\mathbb{C}^{N}\right)^{\ell}, \\
& e_{3}(x)=\left(\begin{array}{c}
1-\left|\mathrm{u}_{1}\right|_{\mathrm{W}}^{2} \\
\vdots \\
1-\left|\mathrm{u}_{\ell}\right|_{\mathrm{W}}^{2}
\end{array}\right) \in \mathbb{R}^{\ell}
\end{aligned}
$$


for $x=(\eta, \mathrm{f}, \psi, \lambda) \in \mathcal{X}$. To write the inequality constraints $f_{a} \leq \mathrm{f}_{j} \leq f_{b}, 1 \leq j \leq \mathrm{n}$, in a compact form, let $g=\left(g_{1}, g_{2}\right): X \rightarrow \mathbb{R}^{2 \mathrm{n}}$ be defined as

$$
g_{1}(x)=\left(\begin{array}{c}
f_{a}-\mathrm{f}_{1} \\
\vdots \\
f_{a}-\mathrm{f}_{\mathrm{n}}
\end{array}\right) \in \mathbb{R}^{\mathrm{n}} \quad \text { and } \quad g_{2}(x)=\left(\begin{array}{c}
\mathrm{f}_{1}-f_{b} \\
\vdots \\
\mathrm{f}_{\mathrm{n}}-f_{b}
\end{array}\right) \in \mathbb{R}^{\mathrm{n}} .
$$

Now we can express the minimization problem as

$$
\min J(x) \text { s.t. } \quad x \in X_{a d},
$$

where the feasible set for $\mathbf{P}$ is given by

$$
x_{a d}=\left\{x \in X \mid e(x)=0 \text { in } y \text { and } g(x) \leq 0 \text { in } \mathbb{R}^{2 n}\right\} .
$$

Summarizing, in addition to given frequencies $\left\{f_{j}\right\}_{j=1}^{n}$ we determine further $\mathrm{n}$ snapshot locations $\left\{\mathrm{f}_{k}\right\}_{k=1}^{\mathrm{n}}$ in an optimal manner by solving $\mathbf{P}$ so that the resulting reduced-order model is improved.

Theorem 4.1. Let Assumption 1 hold. Then, $\mathrm{P}$ has at least one (global) optimal solution $x^{*} \in \mathcal{X}_{a d}$.

Proof. The proof follows by similar arguments as in the proof of Proposition 2.2 in [15]. In particular, we can utilize that the linear and bounded operator $\mathcal{R}(\mathrm{f})$ is defined on the finite dimensional space $\mathbb{C}^{N}$.

4.2. First-order necessary optimality conditions. In this section we present the first-order necessary optimality conditions for $\mathbf{P}$. For the proof we refer the reader to the appendix.

Theorem 4.2. Suppose that Assumption 1 holds. Let $x^{*}=\left(\eta^{*}, \mathrm{f}^{*}, \mathrm{u}^{*}, \lambda^{*}\right) \in X_{a d}$ be a local solution to $\mathbf{P}$. We assume that the eigenvalues $\left\{\lambda_{i}^{*}\right\}_{i=1}^{\ell}$ of $\mathcal{R}\left(\mathrm{f}^{*}\right)$ are simple. Then there exists an associated Lagrange multiplier $\xi^{*}=\left(\mathrm{q}^{*}, \mu^{*}, \phi^{*}\right) \in \mathrm{y}$ satisfying together with $x^{*}$ the following system of adjoint equations:

$$
\begin{array}{ll}
\mathrm{B}_{f}^{\ell, \mathrm{H}} \mathrm{q}^{*}(f)=\left(\begin{array}{c}
\left\langle\mathrm{p}(f)-\mathrm{p}^{\ell, *}(f), \overline{\mathrm{u}}_{1}^{*}\right\rangle_{\mathrm{W}} \\
\vdots \\
\left\langle\mathrm{p}(f)-\mathrm{p}^{\ell, *}(f), \overline{\mathrm{u}}_{\ell}^{*}\right\rangle_{\mathrm{W}}
\end{array}\right), & f \in \mathcal{F}, \\
\left(\mathcal{R}\left(\mathrm{f}^{*}\right)-\lambda_{i}^{*}\right) \mu_{i}^{*}=-\left(\mathrm{G}_{i}^{*}-\left\langle\mathrm{G}_{i}^{*}, \overline{\mathrm{u}}_{i}^{*}\right\rangle_{\mathrm{W}} \mathrm{u}_{i}^{*}\right) \in \operatorname{span}\left\{\mathrm{u}_{i}^{*}\right\}^{\perp} & 1 \leq i \leq \ell, \\
\Re e\left(\left\langle\mathrm{u}_{i}^{*}, \bar{\mu}_{i}^{*}\right\rangle_{\mathrm{W}}\right)=\Re e\left(\left\langle\mathrm{u}_{i}^{*}, \bar{\mu}_{i}^{*}\right\rangle_{\mathrm{W}}\right)=0, & 1 \leq i \leq \ell,
\end{array}
$$

where $\mathrm{p}^{\ell, *}(f)=\sum_{j=1}^{\ell} \eta_{j}^{*}(f) \mathrm{u}_{i}^{*}, \mathrm{q}^{\ell, *}(f)=\sum_{j=1}^{\ell} \mathrm{q}_{j}^{*}(f) \mathrm{u}_{i}^{*}, \mathrm{~B}_{f}^{\ell, \mathrm{H}}=\overline{\mathrm{B}}_{f}^{\ell, \top}, \mathrm{G}_{i}^{*}=$ $\mathrm{W}^{-1} \overline{\mathrm{g}}_{i}^{*}, 1 \leq i \leq \ell$ and

$$
\mathrm{g}_{i}^{*}=\int_{\mathcal{F}} \mathrm{q}_{i}^{*}(f)\left(\overline{\left.\mathrm{B}_{f} \mathrm{p}^{\ell, *}(f)-\mathrm{s}_{f}\right)}+\eta_{i}^{*}(f) \overline{\left(\mathrm{B}_{f}^{\mathrm{H}} \mathrm{q}^{\ell, *}(f)-\frac{\beta}{2} \mathrm{~W}\left(\mathrm{p}(f)-\mathrm{p}^{\ell, *}(f)\right)\right.} \mathrm{d} f\right.
$$

with $\mathrm{B}_{f}^{\mathrm{H}}=\overline{\mathrm{B}}_{f}^{\top}$. Moreover, the following variational inequality holds for $k=1, \ldots, \mathrm{n}$

$$
2 \Re e\left(\sum_{i=1}^{\ell}\left(\left\langle\overline{\mathrm{u}}_{i}^{*}, \dot{\mathrm{p}}_{\mathrm{f}_{k}}^{*}\right\rangle_{\mathrm{W}}\left\langle\overline{\mathrm{p}}_{\mathrm{f}_{k}}^{*}, \mu_{i}^{*}\right\rangle_{\mathrm{W}}+\left\langle\overline{\mathrm{u}}_{i}^{*}, \mathrm{p}_{\mathrm{f}_{k}}^{*}\right\rangle_{\mathrm{W}}\left\langle\dot{\overline{\mathrm{p}}}_{\mathrm{f}_{k}}^{*}, \mu_{i}^{*}\right\rangle_{\mathrm{W}}\right)\left(f-\mathrm{f}_{k}\right)\right) \geq 0
$$

for all $f \in \mathcal{F}$.

The proof of the first-order optimality condition for $\mathbf{P}$ is based on the Wirtinger calculus approach; see, e.g., [2, 21]. 
Remark 4.3. 1) By assumption, all eigenvalues $\left\{\lambda_{i}^{*}\right\}_{i=1}^{\ell}$ of $\mathcal{R}\left(\mathrm{f}^{*}\right)$ are simple. Thus, $\left\langle\mathrm{u}_{i}, \overline{\mathrm{u}}_{j}\right\rangle_{\mathrm{W}}=\delta_{i j}$ holds for $1 \leq i, j \leq \ell$. For $i \in\{1, \ldots, \ell\}$ we decompose $\mu_{i}^{*}$ as follows:

$$
\mu_{i}^{*}=\mu_{i, 1}^{*}+\mu_{i, 2}^{*} \in \operatorname{span}\left\{\mathrm{u}_{i}^{*}\right\}^{\perp} \oplus \operatorname{span}\left\{\mathrm{u}_{i}^{*}\right\}
$$

with $\mu_{i, 1}^{*}=\mu_{i}^{*}-\left\langle\mu_{i}^{*}, \overline{\mathrm{u}}_{i}^{*}\right\rangle_{\mathrm{W}} \mathrm{u}_{i}^{*}$ and $\mu_{i, 2}^{*}=\left\langle\mu_{i}^{*}, \overline{\mathrm{u}}_{i}^{*}\right\rangle_{\mathrm{W}} \mathrm{u}_{i}^{*}$. From

$$
\left(\mathcal{R}\left(\mathrm{f}^{*}\right)-\lambda_{i}^{*}\right) \mu_{i}^{*}=\left(\mathcal{R}\left(\mathrm{f}^{*}\right)-\lambda_{i}^{*}\right) \mu_{i, 1}^{*}
$$

and $4.4 \mathrm{~b}$ we infer that $\mu_{i, 2}^{*}=0$ holds. Thus,

$$
\left\langle\mu_{i}^{*}, \overline{\mathrm{u}}_{i}^{*}\right\rangle_{\mathrm{W}}=0 \text { for } i=1, \ldots, \ell .
$$

2) Note that $4.4 \mathrm{~b}$ is a problem of the size $N$. Therefore, we make use of part 1) to compute an approximation $\mu_{i}^{\ell, *}$ for $\mu_{i}^{*}, 1 \leq i \leq \ell$, by the following projection:

$$
\mu_{i}^{*}=\mu_{i}^{*}-\left\langle\mu_{i}^{*}, \overline{\mathrm{u}}_{i}^{*}\right\rangle_{\mathrm{W}} \mathrm{u}_{i}^{*} \approx \sum_{j=1, j \neq i}^{\ell_{\max }} \gamma_{j}^{*} \mathrm{u}_{j}^{*}=: \mu_{i}^{\ell, *},
$$

where $\left\{\mathrm{u}_{i}^{*}\right\}_{i=1}^{\ell_{\max }}$ are the eigenvectors of $\mathcal{R}\left(\mathrm{f}^{*}\right)$ associated with the eigenvalues $\lambda_{1}^{*}>\ldots>\lambda_{\ell_{\max }^{*}}^{*}>0$ with $\ell_{\max } \in\left\{\ell+1, \ldots, d^{N}\right\}$. To determine the $\gamma_{j}$ 's we

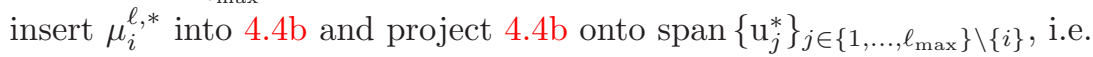

$$
\bar{\Psi}_{i}^{\top}\left(\mathcal{R}\left(\mathrm{f}^{*}\right)-\lambda_{i}^{*}\right) \mu_{i}^{\ell, *}=-\bar{\Psi}_{i}^{\top}\left(\mathrm{G}_{i}^{*}-\left\langle\mathrm{G}_{i}^{*}, \overline{\mathrm{u}}_{i}^{*}\right\rangle_{\mathrm{W}} \mathrm{u}_{i}^{*}\right) \in \mathbb{C}^{\ell_{\max }-1},
$$

where we have set

$$
\Psi_{i}=\left(\mathrm{u}_{1}^{*}|\ldots| \mathrm{u}_{i-1}^{*}\left|\mathrm{u}_{i+1}^{*}\right| \ldots \mid \mathrm{u}_{\ell_{\max }^{*}}^{*}\right) \in \mathbb{C}^{N \times\left(\ell_{\max }-1\right)}, \quad i=1, \ldots, \ell .
$$

4.3. Reduced control problem. Let Assumption 1 be fulfilled. Suppose that $\mathrm{f}=\left(\mathrm{f}_{1}, \ldots, \mathrm{f}_{\mathrm{n}}\right)$ with $\mathrm{f}_{k} \in \mathcal{F}$ for $1 \leq k \leq \mathrm{n}$. Due to Assumption 1 there exists unique finite element solutions $p_{\mathrm{f}_{k}}^{N}=p^{N}\left(\mathrm{f}_{k}\right)$ for $\mathrm{f}_{k} \in \mathcal{F}, 1 \leq k \leq \mathrm{n}$. Thus, we can determine a POD basis $\left\{\psi_{i}(\mathrm{f})\right\}_{i=1}^{\ell}$ of rank $\ell$ and corresponding eigenvalues $\left\{\lambda_{i}(\mathrm{f})\right\}_{i=1}^{\ell}$ by solving the eigenvalue problem 4.1. We set $\psi(\mathrm{f})=\left(\psi_{i}(\mathrm{f})\right)_{1 \leq i \leq \ell} \in$ $\left(\mathbb{C}^{N}\right)^{\ell}$ and $\lambda(\mathfrak{f})=\left(\lambda_{i}(\mathrm{f})\right)_{1 \leq i \leq \ell} \in \mathbb{R}^{\ell}$. Utilizing the POD basis we derive the reducedorder model 3.4 for the Helmholtz equation. By Assumption 1 there exists a unique $\eta$ solving 3.4 for every $f \in \mathcal{F}$. Since the $\psi_{i}$ 's depend on $\mathrm{f}$, the POD coefficient vector $\eta$ depends on $\mathrm{f}$ as well and we write $\eta(\mathrm{f})$. Summarizing, the variables $\eta(\mathrm{f}), \psi(\mathrm{f})$ and $\lambda(f)$ are uniquely determined for a given vector $f$ of frequencies. Thus, we can introduce the so-called reduced cost functional by

$$
\hat{J}(\mathbf{f})=J(\eta(\mathfrak{f}), \mathbf{f}, \psi(\mathbf{f}), \lambda(\mathbf{f})) \quad \text { for } \mathbf{f}=\left(\mathrm{f}_{k}\right)_{1 \leq k \leq \mathrm{n}} \text { with } \mathrm{f}_{k} \in \mathcal{F} .
$$

Then, we consider the reduced problem

$$
\min \hat{J}(\mathrm{f}) \quad \text { s.t. } \quad\left(\mathrm{f}_{k}\right)_{1 \leq k \leq \mathrm{n}} \text { with } \mathrm{f}_{k} \in \mathcal{F}
$$

which is equivalent to the original problem. It follows from $4.4 \mathrm{~d}$ that the gradient of $\hat{J}$ is given by

$$
\nabla \hat{J}(\mathbf{f})=2 \Re e\left(\begin{array}{c}
\sum_{i=1}^{\ell}\left(\left\langle\bar{\psi}_{i}^{*}, \dot{\mathrm{p}}_{\mathrm{f}_{1}}^{*}\right\rangle_{\mathrm{W}}\left\langle\overline{\mathrm{p}}_{\mathrm{f}_{1}}^{*}, \mu_{i}^{*}\right\rangle_{\mathrm{W}}+\left\langle\bar{\psi}_{i}^{*}, \mathrm{p}_{\mathrm{f}_{1}}^{*}\right\rangle_{\mathrm{W}}\left\langle\dot{\overline{\mathrm{f}}}_{\mathrm{f}_{1}}^{*}, \mu_{i}^{*}\right\rangle_{\mathrm{W}}\right) \\
\vdots \\
\sum_{i=1}^{\ell}\left(\left\langle\bar{\psi}_{i}^{*}, \dot{\mathrm{p}}_{\mathrm{f}_{\mathrm{n}}}^{*}\right\rangle_{\mathrm{W}}\left\langle\overline{\mathrm{p}}_{\mathrm{f}_{\mathrm{n}}}^{*}, \mu_{i}^{*}\right\rangle_{\mathrm{W}}+\left\langle\bar{\psi}_{i}^{*}, \mathrm{p}_{\mathrm{f}_{\mathrm{n}}}^{*}\right\rangle_{\mathrm{W}}\left\langle\dot{\mathrm{p}}_{\mathrm{f}_{\mathrm{n}}}^{*}, \mu_{i}^{*}\right\rangle_{\mathrm{W}}\right)
\end{array}\right) .
$$


4.4. Adaptive POD basis computation. In this section we introduce the adaptive POD basis computation strategy. To start we have a look at the decay of the normalized singular values. In order to have an efficient reduced order model it is required that the singular values decrease fast and hence the number of needed POD basis functions is small. When looking at Figure 4.1 we can see that the normalized singular values do not decrease fast in the beginning. This implies that many

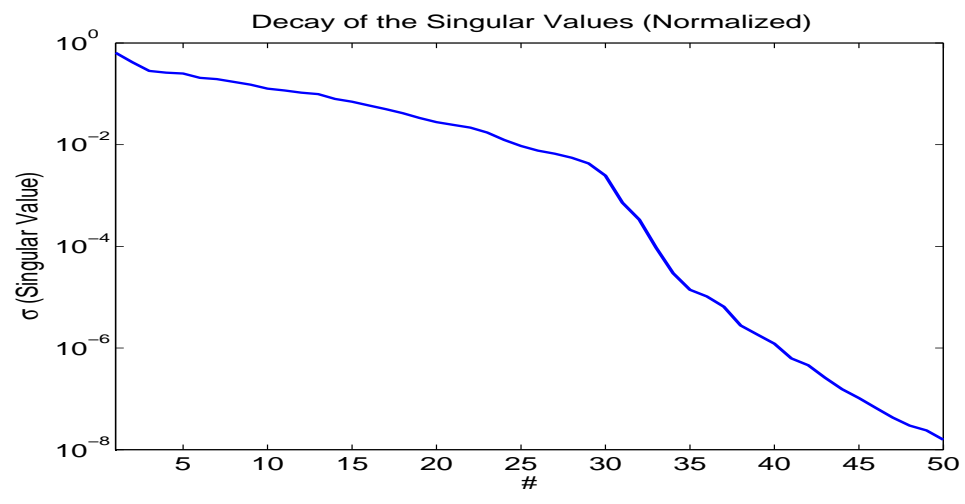

Figure 4.1. Decay of the singular values for the frequency range from 200 to $500 \mathrm{~Hz}$.

POD basis functions will be needed to approximate the frequency range from 200 to $500 \mathrm{~Hz}$. Here one would need approximately 30 POD basis functions. Since the variables are complex this means 30 real and 30 imaginary basis functions. Hence we want to develop a strategy to compute the best possible POD basis with the fewest snapshots required.

Let us now introduce an adaptive strategy. For this we use the obtained results from the optimal snapshot locations. The idea is to start with a small frequency range and extend it iteratively. This can be done a-priori or when it is required by the application. When extending the frequency range we have to solve either the optimization problem $\mathbf{P}$ or $\hat{\mathbf{P}}$ to add the best possible snapshots.

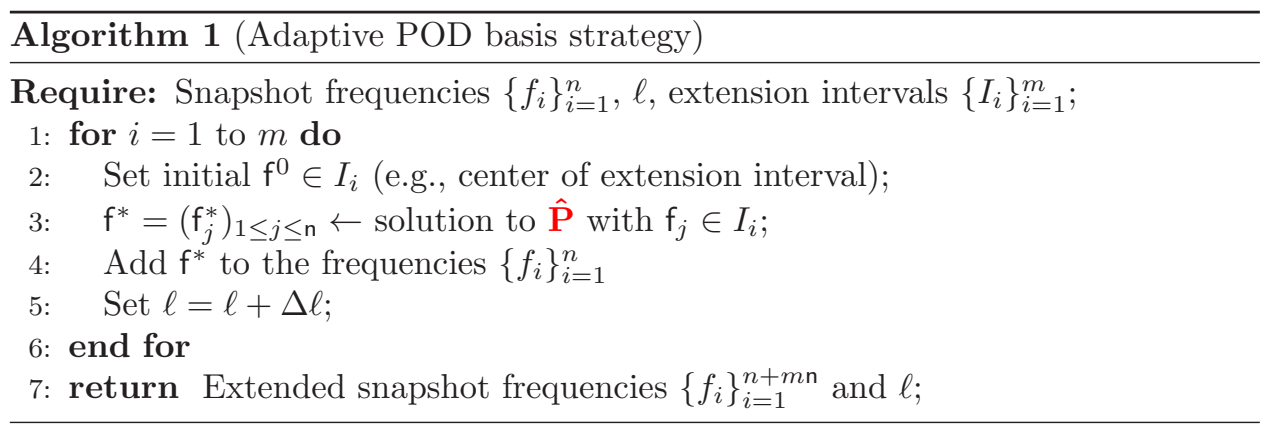

5. Numerical experiments. In this section we will present some numerical results for the adaptive POD approach described in Section 4.4. Let us start with the problem settings. The acoustic domain $\Omega \subset \mathbb{R}^{2}$ and the impedance boundary $\Gamma_{\mathrm{R}}=\left\{\boldsymbol{x}=\left(x_{1}, 0\right) \mid 0.5 \leq x \leq 1.8\right\}$ are illustrated in Figure 5.1. We apply a stan- 

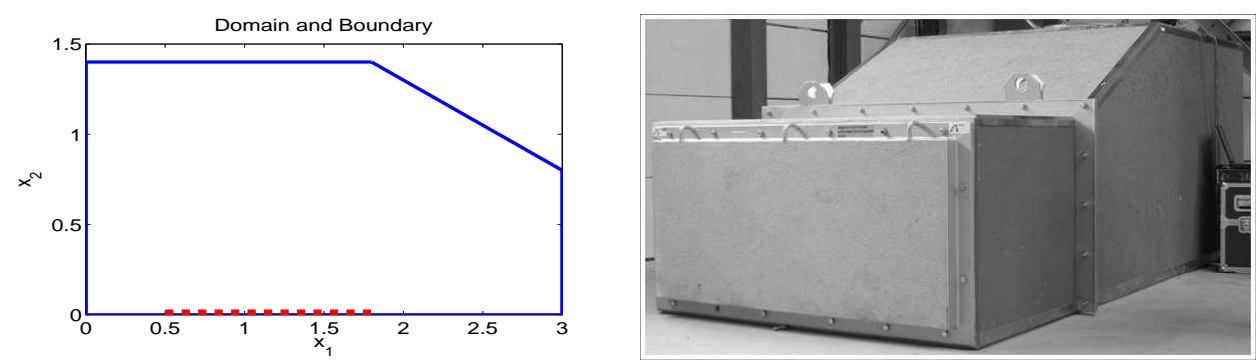

FIGURE 5.1. Left plot: computational domain $\Omega \subset \mathbb{R}^{2}$ together with the boundary $\Gamma_{\mathrm{N}}$ (black line) and $\Gamma_{\mathrm{R}}$ (dashed line); Right plot: experimental design at the Competence Center The Virtuale Vehicle in Graz (see http://www.vif.tugraz.at).

dard piecewise linear finite element (FE) discretization with 695 degrees of freedom. Next let us introduce the missing parameters. The right hand side is given as

$$
s_{f}(\boldsymbol{x})=\frac{1}{5} \exp \left(\frac{\jmath \pi(f-200)}{50}\right) \exp \left(-50\left|\boldsymbol{x}-\boldsymbol{x}_{q}\right|^{2}\right) \quad \text { for } \boldsymbol{x} \in \Omega \text { and } f \in[200,500] \text {, }
$$

where $\boldsymbol{x}_{q}=(0.28,1.21)$. The parameters $c, k_{f}$ and $\omega_{f}$ are already given in 2.7 . Lastly the $f$-dependent admittance $A_{f}$ are plotted in the right plot of Figure 2.1. The weights $\alpha_{j}$ in the optimization $\hat{\mathbf{P}}$ are set to one. Further the scaling parameter $\beta$ is set to

$$
\beta=\sum_{j=1}^{M} \alpha_{j}\left\|p^{N}\left(f_{j}\right)\right\|_{V}^{2} \mathrm{~d} f
$$

with $f_{j}=f_{a}+(j-1) \Delta f, \Delta f=\left(f_{b}-f_{a}\right) / M$ and trapezoidal weights $\left\{\alpha_{j}\right\}_{j=1}^{n}$. All computations are carried out with complex variables, i.e. the real and imaginary parts are not separated. The integral over $\mathcal{F}$ is computed by using a trapezoidal rule, where $\mathcal{F}$ is discretized equidistant with stepsize one.

The optimization problem is solved by using a quasi-Newton method, since the second derivative is rather involved. For the convergence analysis of these methods we refer the reader, e.g., to [18]. The code is written in MatLaB utilizing the Partial Differential Toolbox and the Optimization Toolbox. Hence, for convenience the routine fmincon is used to carry out the optimization. The options are chosen as follows

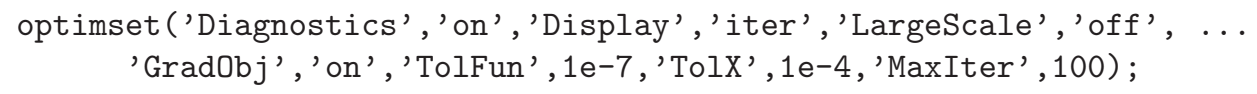

Note that we supply the routine with the analytical gradient 4.6. In the following we will present numerical results for different scenarios using the adaptive POD strategy.

Run 1 (One optimal snapshot location for a fixed interval). We consider the frequency range from 300 to $330 \mathrm{~Hz}$. Given the snapshots to the frequencies $f_{i}=300+i$ for $i=0, \ldots, 15$. In this test we want to find one additional frequency $\mathrm{f}$ to minimize the cost given by 4.3 , i.e., we have $\mathrm{n}=1$. We take $\ell=4$ POD basis functions. In the left plot of Figure 5.2 (left) the behaviour of the cost function is presented for three different scenarios. The dash-dotted line represents the case when no additional snapshot is added, i.e. the snapshots corresponding to the frequencies 

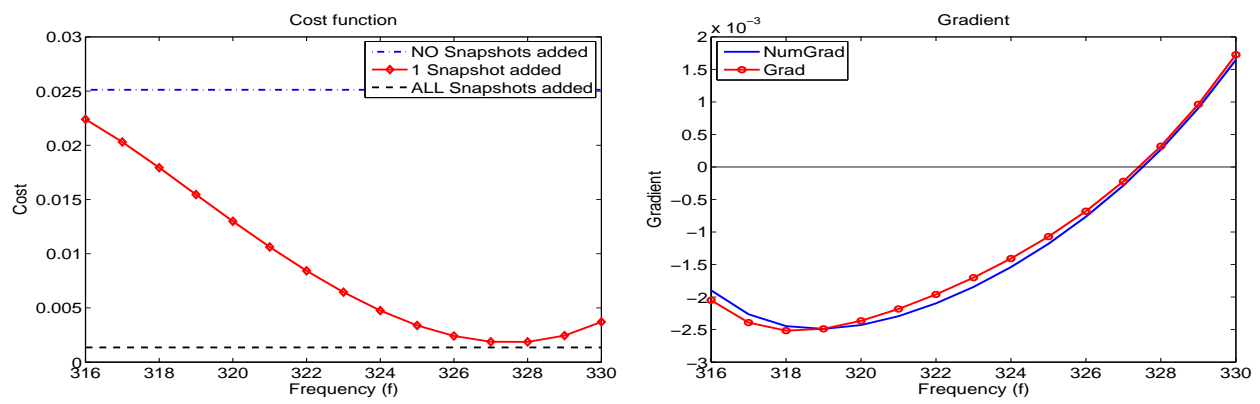

Figure 5.2. Run 1: behaviour of the cost function for three different scenarios (left plot) and the corresponding gradient $(\nabla \hat{J}(\mathbf{f}))$ (right plot).

$f_{i}=300+i, i=0, \ldots, 15$, are used to compute the POD basis of rank $\ell$. The dashed line represents the case that the POD basis of rank $\ell$ is computed using snapshots corresponding to $f_{i}=300+i, i=0, \ldots, 30$, i.e. all the frequencies. The line with stars represents the addition of a single snapshot corresponding to frequency $f \in[315,330]$. It can be seen that by adding a single snapshot nearly the same value for the cost function can be reached compared to adding snapshots for all the frequencies. In the right plot of Figure 5.2 a comparison of the numerical (NumGrad) and the analytical (Grad) gradient are plotted. In Table 5.2 we compare the obtained optimal $f$ when using the analytical and numerical gradient and the performance of the optimization algorithm. It can be seen that for both

\begin{tabular}{lcccc}
\hline Method & $f^{*}$ & $\hat{J}\left(f^{*}\right)$ & CPU time & iterations \\
\hline Grad & 327.44 & 0.001804 & 0.95 & 6 \\
NumGrad & 327.53 & 0.001802 & 0.62 & 6 \\
\hline
\end{tabular}

TABLE 5.2. Run 1: results for adding one additional snapshot by optimization using the analytical gradient (Grad) and the numerical approximation of the gradient (NumGrad).

cases similar results are obtained. The optimal frequency $\mathrm{f}^{*}$ is around $327.5 \mathrm{~Hz}$ and the cost function has the approximate value 0.0018. For both types of gradients the optimization method needs the same number of iterations. The optimization utilizing both analytical and numerical gradient are comparable. For comparison, the value of the cost function using the frequencies $f_{i}=300+i$ for $i=0, \ldots, 30$ is 0.0014 , which is a very small difference.

Run 2 (Two optimal snapshot locations for a fixed interval). In this experiment we consider the same settings as in Run 1 . The main difference is that we now want to add not only one new snapshot but two. Hence we will be looking for a tuple $\mathrm{f}=$ $\left(\mathrm{f}_{1}, \mathrm{f}_{2}\right) \in[316,330] \times[316,330]$ to minimize the cost function 4.3. First we again look at the evaluation of the cost function for all the different combinations for $f=\left(f_{1}, f_{2}\right)$. Figure 5.3 shows the evaluation of the cost function as $3 \mathrm{D}$ plot and as contour plot. It can be seen that the cost function is symmetric, i.e. $\hat{J}\left(f_{1}, f_{2}\right)=\hat{J}\left(f_{2}, f_{1}\right)$. Further in Figure 5.4 a comparison of the analytical and numerical gradient fields is shown. 

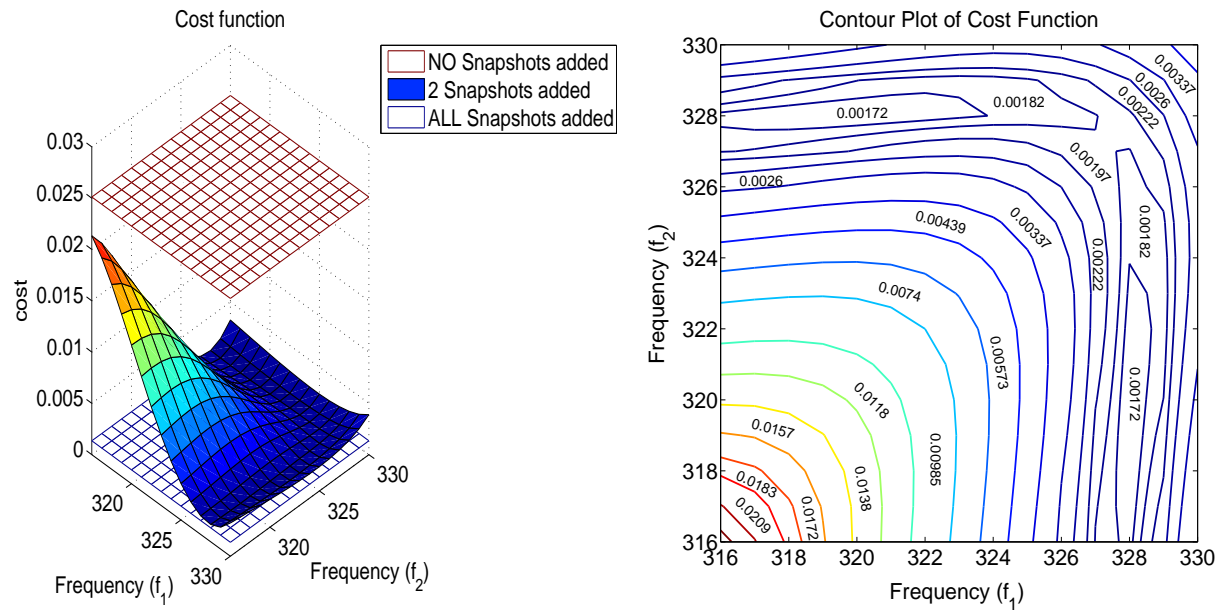

Figure 5.3. Run 2: cost function when adding two additional snapshots $\left(\mathrm{f}_{1}, \mathrm{f}_{1}\right)$ in $3 \mathrm{D}$ (left plot) and as contour plot (right plot).

Next we look at the optimal $f^{*}$ obtained by the optimization, see Table 5.4. Again
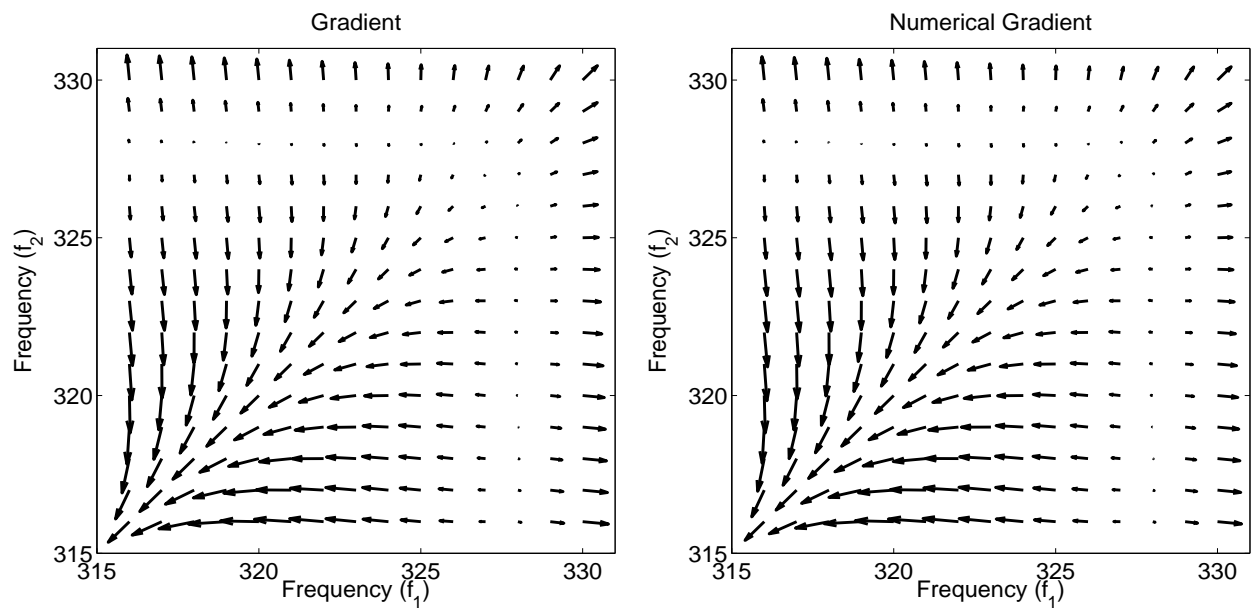

Figure 5.4. Run 2: analytical (left plot) and numerical (right plot) gradient $\nabla \hat{J}(\mathrm{f})$ when adding two additional snapshots $\mathrm{f}=$ $\left(f_{1}, f_{1}\right)$.

we observe that using the analytical and numerical gradient deliver very similar results. The obtained result for the frequencies is around $\mathrm{f}^{*}=(319.85,328.10)$ and the corresponding value of the cost function is at 0.0016 . Recall that the value of the cost function using the whole frequency range is 0.0014 and for adding only one additional frequency is 0.0018 . So there is a small improvement when adding two frequencies. Comparing the CPU-time needed for the optimization there is no large difference. As with adding one frequency the optimization utilizing different gradients are again comparable. 


\begin{tabular}{lccccc}
\hline Method & $\mathrm{f}_{1}^{*}$ & $\mathrm{f}_{2}^{*}$ & $\hat{J}\left(\mathrm{f}^{*}\right)$ & CPU time & Iterations \\
\hline Grad & 319.86 & 328.07 & 0.001610 & 0.66 & 5 \\
NumGrad & 319.84 & 328.15 & 0.001608 & 0.74 & 6 \\
\hline
\end{tabular}

TABLE 5.4. Run 2: results for adding two additional snapshots by optimization using the analytical gradient (Grad) and the numerical approximation of the gradient (NumGrad).

Run 3 (Adaptive POD basis computation). In this experiment we focus on the successive adding of additional frequencies. For this we consider the following settings. We use an initial frequency range $f_{i}=199+i \in[200,210]$ for $i=1, \ldots, 11$. And we initially set $\ell=3$. Now we want to construct a POD basis for the frequency range $[200,300]$ using the strategy described by Algorithm 1. For this we introduce the extension intervals $I_{i}=[200+10 i, 200+10(i+1)]$ for $i=1, \ldots, m$ with $m=9$. In each interval we will compute an optimal $\mathrm{f}_{I_{i}}^{*}$ and add it to the frequency set $\left\{f_{1}, \ldots, f_{11}, f_{I_{1}}^{*}, \ldots, f_{I_{i-1}}^{*}\right\}$. The number of POD basis functions is increased alternately, i.e. in the intervals with even index we increase $\ell$ by $\Delta \ell=1$ and the intervals with odd index we do not increase $\ell$. Thus, we will utilize $\ell=8$ POD basis functions for the computation of $f_{I_{9}}^{*}$ in the last interval $I_{9}$. This is realized by the MATLAB command $e l l=e l l+\bmod (i, 2)$, where $i$ is the index for the intervals. As the initial guess $\mathrm{f}_{I_{i}}^{0}$ for $\mathrm{f}_{I_{i}}^{*}$ in each interval we choose the corresponding midpoints. In Table 5.4 the initial and added snapshot for each interval $I_{i}$ is shown. Again

\begin{tabular}{lccc}
\hline Method & Grad & NumGrad & \\
\hline Interval $I_{i}$ & $\mathrm{f}_{I_{i}}^{*}$ & $\mathrm{f}_{I_{i}}^{*}$ & initial $\mathrm{f}_{I_{i}}^{0}$ \\
\hline$I_{1}=[210,220]$ & 218.1510 & 218.1684 & 215 \\
$I_{2}=[220,230]$ & 224.9994 & 223.9296 & 225 \\
$I_{3}=[230,240]$ & 238.2373 & 238.1699 & 235 \\
$I_{4}=[240,250]$ & 246.8142 & 247.0282 & 245 \\
$I_{5}=[250,260]$ & 255.8309 & 255.5789 & 255 \\
$I_{6}=[260,270]$ & 265.8839 & 265.9337 & 265 \\
$I_{7}=[270,280]$ & 270.0000 & 280.0000 & 275 \\
$I_{8}=[280,290]$ & 285.0000 & 290.0000 & 285 \\
$I_{9}=[290,300]$ & 293.7210 & 293.9376 & 295 \\
\hline
\end{tabular}

TABLE 5.4. Run 3: initial and optimal frequency for each interval obtained by the optimization using the analytical gradient (Grad) and numerical gradient (NumGrad).

we compare the results when using an analytical gradient to the results obtained when using a numerical approximation of the gradient. One can observe that the obtained $f$ in each interval are very similar. In the intervals $I_{7}$ and $I_{8}$ a larger difference can be observed. This is due to different optimization strategies used by MATLAB and an almost constant cost function. To compare the performance of the adaptive strategy we look at the value of the cost function 4.3 as well as the 
approximation error

$$
\operatorname{Err}=\sum_{f=200}^{300}\left\|p^{N}(f)-p^{\ell}(f)\right\|_{V}^{2}=\sum_{f=200}^{300}\left|\mathrm{p}(f)-\mathrm{p}^{\ell}(f)\right|_{\mathrm{W}}^{2}=\sum_{f=200}^{300}|\mathrm{p}(f)-\Psi \eta(f)|_{\mathrm{W}}^{2} .
$$

It is desired that Err is sufficiently small. We define the sets

$$
\begin{array}{ll}
\mathfrak{F}_{n}=\left\{f_{1}, \ldots, f_{11}\right\}, & \mathfrak{F}^{*}=\left\{\mathrm{f}_{I_{1}}^{*}, \ldots \mathrm{f}_{I_{9}}^{*},\right. \\
\mathfrak{F}_{\text {all }}=\mathfrak{F}_{n} \cup\{211,212, \ldots, 300\}, & \mathfrak{F}_{\text {init }}=\left\{\mathrm{f}_{I_{1}}^{0}, \ldots, \mathrm{f}_{I_{9}}^{0}\right\} .
\end{array}
$$

Additionally to adding the optimal snapshots we compare the following scenarios:

1) use snapshots at the frequencies $\mathfrak{F}_{n}$ to get a POD basis of rank three,

2) utilize snapshots at the frequencies $\mathfrak{F}_{n} \cup \mathfrak{F}_{\text {init }}$ to compute a POD basis of rank eight,

3) use snapshots at the frequencies $\mathfrak{F}_{\text {all }}$ to determine a POD basis of rank four,

4) utilize snapshots at the frequencies $\mathfrak{F}_{\text {all }}$ to compute a POD basis of rank eight.

These scenarios are interesting since from the results it can be seen whether the adaptive POD strategy is effective. The question is if it is necessary to add addi-

\begin{tabular}{|c|c|c|c|c|c|}
\hline Method & & Grad & NumGrad & Grad & NumGrad \\
\hline Frequencies & $\ell$ & \multicolumn{2}{|c|}{$\hat{J}\left(\mathrm{f}^{*}\right)$} & \multicolumn{2}{|c|}{ Err } \\
\hline $\mathfrak{F}_{n}$ & 3 & \multicolumn{2}{|c|}{5.2952} & \multicolumn{2}{|c|}{0.1662} \\
\hline $\mathfrak{F}_{\text {all }}$ & 4 & \multicolumn{2}{|c|}{2.3517} & \multicolumn{2}{|c|}{0.0738} \\
\hline $\mathfrak{F}_{n} \cup \mathfrak{F}_{\text {init }}$ & 8 & \multicolumn{2}{|c|}{0.2717} & \multicolumn{2}{|c|}{0.0085} \\
\hline $\mathfrak{F}_{n} \cup \mathfrak{F}^{*}$ & 8 & 0.0234 & 0.0230 & 0.0007 & 0.0007 \\
\hline $\mathfrak{F}_{\text {all }}$ & 8 & \multicolumn{2}{|c|}{0.0550} & \multicolumn{2}{|c|}{0.0017} \\
\hline
\end{tabular}
tional snapshots or if adding the center of an extension interval is also sufficient. In Table 5.4 the results are summarized. It can be seen that the adaptive strategy pro-

TABLE 5.4. Run 3: comparison of the results of different scenarios for the computation of the POD basis. The choice $\mathfrak{F}_{n} \cup \mathfrak{F}^{*}$ in the forth row correspond to the adaptive POD basis approach of Algorithm 1 using the analytical gradient (Grad) and the numerical approximation of the gradient (NumGrad).

vides the lowest value in the cost function and the lowest approximation error. Even the approximation error obtained by using $\mathfrak{F}_{\text {all }}$ is larger. This can be explained by the fact that by the optimization the optimal snapshots are added. Hence snapshots are obtained which are not in the set $\mathfrak{F}_{\text {all }}$. Not adding any additional snapshot $\left(\mathfrak{F}_{n}\right)$ or just adding the centers of the extension intervals $\left(\mathfrak{F}_{n} \cup \mathfrak{F}_{\text {init }}\right)$ lead to significant larger values for the cost function as well as the approximation error. Further we can see that using the analytical gradient or the numerical gradient deliver almost the same values for the cost function and approximation error although some differences in the optimal frequencies could be observed in Table 5.4. To conclude this numerical experiment we look at the performance of the two approaches in the means of computational time. When using the analytical gradient the adaptive strategy needs 33.9 seconds to terminate. Compared to the numerical gradient which needs 12.2 seconds this is longer but the algorithm can not be compared directly since the 
MATLAB routine internally use slightly different methods. What can be said is that the strategy is applicable since the computational efforts are not too large and the obtained optimal frequencies lead to better snapshots and hence to a better POD basis. Further this strategy can directly be incorporated with the application. The extension of the interval can be performed when required and with the strategy it is guaranteed that the extension is performed in an optimal way.

\section{Appendix.}

Proof of Theorem 4.2. The existence of a unique Lagrange multiplier $\xi^{*}=$ $\left(\mathrm{q}^{*}, \mu^{*}, \phi^{*}\right) \in \mathrm{y}$ follows from a standard constraint qualification; see, e.g., [17]. The application for the present application is analogous to the proof of Proposition 2.4 in [15]. Here, we use that, by Assumption 1, both 2.13 and 2.15 are uniquely solvable for any $f \in \mathcal{F}$; see Remark 2.5 and Corollary 2.6.

To derive the first-order optimality condition for $\mathbf{P}$ we apply the Wirtinger calculus approach $[2,21]$. For this we introduce the Lagrange functional $\mathcal{L}$ : $x \times x \times y \times y \rightarrow \mathbb{C}$ is defined as:

$$
\begin{aligned}
\mathcal{L}(\zeta)= & \frac{\beta}{2} \int_{\mathcal{F}}\left\langle\mathrm{p}(f)-\sum_{i=1}^{\ell} \eta_{i}(f) \mathrm{u}_{i}, \overline{\mathrm{p}}(f)-\sum_{j=1}^{\ell} \bar{\eta}_{j}(f) \overline{\mathrm{u}}_{j}\right\rangle_{\mathrm{W}} \mathrm{d} f \\
& +\int_{\mathcal{F}}\left(\overline{\mathrm{B}_{f}^{\ell} \eta(f)-\mathrm{s}_{f}^{\ell}}\right)^{\top} \mathrm{q}(f)+\left(\mathrm{B}_{f}^{\ell} \eta(f)-\mathrm{s}_{f}^{\ell}\right)^{\top} \tilde{\mathrm{q}}(f) \mathrm{d} f \\
& +\sum_{i=1}^{\ell}\left(\left\langle\left(\overline{\mathcal{R}(\mathrm{f})-\lambda_{i}}\right) \overline{\mathrm{u}}_{i}, \mu_{i}\right\rangle_{\mathrm{W}}+\left\langle\left(\mathcal{R}(\mathrm{f})-\lambda_{i}\right) \mathrm{u}_{i}, \tilde{\mu}_{i}\right\rangle_{\mathrm{W}}+\left(1-\left|\mathrm{u}_{i}\right|_{\mathrm{W}}^{2}\right) \phi_{i}\right)
\end{aligned}
$$

for $\zeta=(z, \bar{z}, \xi, \tilde{\xi}), z=(\eta, \mathrm{f}, \mathrm{u}, \lambda) \in X$ and $\xi=(\mathrm{q}, \mu, \phi), \tilde{\xi}=(\tilde{\mathrm{q}}, \tilde{\mu}, \phi) \in \mathrm{y}$. We will show that the Lagrange multiplier $\xi^{*}$ associated with the optimal solution $z^{*}$ satisfies $\bar{\xi}^{*}=\tilde{\xi}^{*}$. Since $1-\left|\mathrm{u}_{i}\right|_{W}^{2} \in \mathbb{R}$ holds, we use a standard Lagrange multiplier for this constraint.

First we compute the derivative of the Lagrangian with respect to $\eta$ in an arbitrary direction $\eta_{\delta}=\left(\eta_{\delta, 1}, \ldots, \eta_{\delta, \ell}\right) \in H^{1}\left(\mathcal{F} ; \mathbb{C}^{\ell}\right)$. We derive that

$$
\mathcal{L}_{\eta}(\zeta) \eta_{\delta}=\int_{\mathcal{F}}\left(\left(\mathrm{B}_{f}^{\ell} \eta_{\delta}(f)\right)^{\top} \tilde{\mathrm{q}}(f)-\frac{\beta}{2} \sum_{i=1}^{\ell} \eta_{\delta, i}(f)\left\langle\mathrm{u}_{i}, \overline{\mathrm{p}}(f)-\sum_{j=1}^{\ell} \bar{\eta}_{j}(f) \overline{\mathrm{u}}_{j}\right\rangle_{\mathrm{W}}\right) \mathrm{d} f .
$$

Setting $\mathbf{B}_{f}^{\ell, \top}=\left(\mathrm{B}_{f}^{\ell}\right)^{\top}$ we have

$$
\left(\mathrm{B}_{f}^{\ell} \eta_{\delta}(f)\right)^{\top} \tilde{\mathrm{q}}(f)=\eta_{\delta}(f)^{\top} \mathrm{B}_{f}^{\ell, \top} \tilde{\mathrm{q}}(f)=\sum_{i=1}^{\ell} \eta_{\delta, i}(f)\left(\mathrm{B}_{f}^{\ell, \top} \tilde{\mathrm{q}}(f)\right)_{i}
$$

for $f \in \mathcal{F}$. Thus, it follows that

$$
\mathcal{L}_{\eta}(\zeta) \eta_{\delta}=\int_{\mathcal{F}} \sum_{i=1}^{\ell} \eta_{\delta, i}(f)\left(\left(\mathrm{B}_{f}^{\ell, \top} \tilde{\mathrm{q}}(f)\right)_{i}-\frac{\beta}{2}\left\langle\mathrm{u}_{i}, \overline{\mathrm{p}}(f)-\sum_{j=1}^{\ell} \bar{\eta}_{j}(f) \overline{\mathrm{u}}_{j}\right\rangle_{\mathrm{W}}\right) \mathrm{d} f
$$

for all $\eta_{\delta} \in H^{1}\left(\mathcal{F} ; \mathbb{C}^{\ell}\right)$. Therefore, the first-order necessary optimality condition $\mathcal{L}_{\eta}\left(\zeta^{*}\right) \eta_{\delta}=0$ in $H^{1}\left(\mathcal{F} ; \mathbb{C}^{\ell}\right)^{\prime}$ at $\zeta^{*}=\left(z^{*}, \bar{z}^{*}, \xi^{*}, \tilde{\xi}^{*}\right)$ implies that

$$
\mathrm{B}_{f}^{\ell, \top} \tilde{\mathrm{q}}^{*}(f)=\frac{\beta}{2}\left(\begin{array}{c}
\left\langle\mathrm{u}_{1}^{*}, \overline{\mathrm{p}}(f)-\overline{\mathrm{p}}^{\ell, *}(f)\right\rangle_{\mathrm{W}} \\
\vdots \\
\left\langle\mathrm{u}_{\ell}^{*}, \overline{\mathrm{p}}(f)-\overline{\mathrm{p}}^{\ell, *}(f)\right\rangle_{\mathrm{W}}
\end{array}\right) \quad \text { for } f \in \mathcal{F},
$$


where we have set $\overline{\mathrm{p}}^{\ell, *}(f)=\sum_{j=1}^{\ell} \bar{\eta}_{j}^{*}(f) \overline{\mathrm{u}}_{j}^{*}$.

Next we turn to the derivative of the Lagrangian with respect to $\bar{\eta}$ and derive

$$
\mathcal{L}_{\bar{\eta}}(\zeta) \tilde{\eta}_{\delta}=\int_{\mathcal{F}}\left(\overline{\mathrm{B}}_{f}^{\ell} \tilde{\eta}_{\delta}(f)\right)^{\top} \mathrm{q}(f)-\frac{\beta}{2} \sum_{i=1}^{\ell} \tilde{\eta}_{\delta, i}(f)\left\langle\mathrm{p}(f)-\sum_{j=1}^{\ell} \eta_{j}(f) \mathrm{u}_{j}, \overline{\mathrm{u}}_{i}\right\rangle_{\mathrm{W}} \mathrm{d} f
$$

for all $\tilde{\eta}_{\delta}=\left(\tilde{\eta}_{\delta, 1}, \ldots, \tilde{\eta}_{\delta, \ell}\right) \in H^{1}\left(\mathcal{F} ; \mathbb{C}^{\ell}\right)$. Notice that

$$
\left(\overline{\mathrm{B}}_{f}^{\ell} \tilde{\eta}_{\delta}(f)\right)^{\top} \mathrm{q}(f)=\tilde{\eta}_{\delta}(f)^{\top} \overline{\mathrm{B}}_{f}^{\ell, \top} \mathrm{q}(f)=\tilde{\eta}_{\delta}(f)^{\top} \mathrm{B}_{f}^{\ell, \mathrm{H}} \mathrm{q}(f)=\sum_{i=1}^{\ell} \tilde{\eta}_{\delta, i}(f)\left(\mathrm{B}_{f}^{\ell, \mathrm{H}} \mathrm{q}(f)\right)_{i}
$$

for $f \in \mathcal{F}$. Consequently,

$$
\mathcal{L}_{\bar{\eta}}(\zeta) \tilde{\eta}_{\delta}=\int_{\mathcal{F}} \sum_{i=1}^{\ell} \tilde{\eta}_{\delta, i}(f)\left(\left(\mathrm{B}_{f}^{\ell, \mathrm{H}} \mathrm{q}(f)\right)_{i}-\frac{\beta}{2}\left\langle\mathrm{p}(f)-\sum_{j=1}^{\ell} \eta_{j}(f) \mathrm{u}_{j}, \overline{\mathrm{u}}_{i}\right\rangle_{\mathrm{W}}\right) \mathrm{d} f
$$

for all $\tilde{\eta}_{\delta} \in H^{1}\left(\mathcal{F} ; \mathbb{C}^{\ell}\right)$. Thus, the first-order necessary optimality condition $\mathcal{L}_{\bar{\eta}}\left(\zeta^{*}\right)=$ 0 in $H^{1}\left(\mathcal{F} ; \mathbb{C}^{\ell}\right)^{\prime}$ implies that

$$
\mathrm{B}_{f}^{\ell, \mathrm{H}} \mathrm{q}^{*}(f)=\frac{\beta}{2}\left(\begin{array}{c}
\left\langle\mathrm{p}(f)-\mathrm{p}^{\ell, *}(f), \overline{\mathrm{u}}_{1}^{*}\right\rangle_{\mathrm{W}} \\
\vdots \\
\left\langle\mathrm{p}(f)-\mathrm{p}^{\ell, *}(f), \overline{\mathrm{u}}_{\ell}^{*}\right\rangle_{\mathrm{W}}
\end{array}\right) \quad \text { for } f \in \mathcal{F}
$$

with $\mathrm{p}^{\ell, *}(f)=\sum_{i=1}^{\ell} \eta^{*}(f) \mathrm{u}_{i}^{*}$, which is 4.4 a. Combining A.1 and A.2 we infer that

$$
\begin{aligned}
\mathrm{B}_{f}^{\ell, \mathrm{H}} \mathrm{q}^{*}(f) & =\left(\begin{array}{c}
\left\langle\mathrm{p}(f)-\mathrm{p}^{\ell, *}(f), \overline{\mathrm{u}}_{1}^{*}\right\rangle_{\mathrm{W}} \\
\vdots \\
\left\langle\mathrm{p}(f)-\mathrm{p}^{\ell, *}(f), \overline{\mathrm{u}}_{\ell}^{*}\right\rangle_{\mathrm{W}}
\end{array}\right)=\left(\begin{array}{c}
\overline{\left\langle\mathrm{u}_{1}^{*}, \overline{\mathrm{p}}(f)-\overline{\mathrm{p}}^{\ell, *}(f)\right\rangle_{\mathrm{W}}} \\
\frac{\vdots}{\left\langle\mathrm{u}_{\ell}^{*}, \overline{\mathrm{p}}(f)-\overline{\mathrm{p}}^{\ell, *}(f)\right\rangle_{\mathrm{W}}}
\end{array}\right) \\
= & \overline{\mathrm{B}_{f}^{\ell, \top} \tilde{\mathrm{q}}^{*}(f)}=\mathrm{B}_{f}^{\ell, \mathrm{H}} \overline{\tilde{\mathrm{q}}}^{*}(f) \quad \text { for } f \in \mathcal{F} .
\end{aligned}
$$

It follows from Assumption 1 and Remark 2.5 that the matrix $\mathrm{B}_{f}^{\ell}$ is invertible for all $f \in \mathcal{F}$. Thus we derive that

$$
\overline{\mathrm{q}}^{*}=\tilde{\mathrm{q}}^{*} \quad \text { in } L^{2}\left(\mathcal{F} ; \mathbb{C}^{\ell}\right)
$$

holds, i.e., A.1 and A.2 are equivalent. In the sequel we will replace $\tilde{\mathrm{q}}^{*}$ by $\overline{\mathrm{q}}^{*}$.

Now we consider the derivative of the Lagrangian with respect to $f=\left(f_{1}, \ldots, f_{n}\right)$. For $1 \leq k \leq \mathrm{n}$ it follows that

$$
\frac{\partial \mathcal{R}}{\partial \mathrm{f}_{k}}(\mathrm{f}) \mathrm{u}=\left\langle\mathrm{u}, \dot{\overline{\mathrm{p}}}_{\mathrm{f}_{k}}\right\rangle_{\mathrm{W}} \mathrm{p}_{\mathrm{f}_{k}}+\left\langle\mathrm{u}, \overline{\mathrm{p}}_{\mathrm{f}_{k}}\right\rangle_{\mathrm{W}} \dot{\mathrm{p}}_{\mathrm{f}_{k}} \quad \text { for } \mathrm{u} \in \mathbb{C}^{\ell},
$$


where $\dot{\mathrm{p}}_{\mathrm{f}_{k}}$ solves 2.15 for $f=\mathrm{f}_{k}$. Therefore, we have $\mathcal{L}_{\mathrm{f}_{k}}(\zeta)$

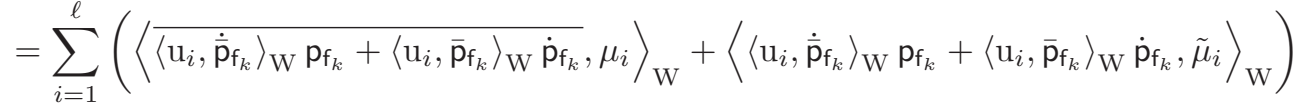

$$
\begin{aligned}
& =\sum_{i=1}^{\ell}\left(\left\langle\left\langle\overline{\mathrm{u}}_{i}, \dot{\mathrm{p}}_{\mathrm{f}_{k}}\right\rangle_{\mathrm{W}} \overline{\mathrm{p}}_{\mathrm{f}_{k}}+\left\langle\overline{\mathrm{u}}_{i}, \mathrm{p}_{\mathrm{f}_{k}}\right\rangle_{\mathrm{W}} \dot{\overline{\mathrm{p}}}_{\mathrm{f}_{k}}, \mu_{i}\right\rangle_{\mathrm{W}}+\left\langle\left\langle\mathrm{u}_{i}, \dot{\overline{\mathrm{f}}}_{\mathrm{f}_{k}}\right\rangle_{\mathrm{W}} \mathrm{p}_{k}+\left\langle\mathrm{u}_{i}, \overline{\mathrm{p}}_{\mathrm{f}_{k}}\right\rangle_{\mathrm{W}} \dot{\mathrm{p}}_{\mathrm{f}_{k}}, \tilde{\mu}_{i}\right\rangle_{\mathrm{W}}\right) \\
& =\sum_{i=1}^{\ell}\left(\left\langle\overline{\mathrm{u}}_{i}, \dot{\mathrm{p}}_{\mathrm{f}_{k}}\right\rangle_{\mathrm{W}}\left\langle\overline{\mathrm{p}}_{\mathrm{f}_{k}}, \mu_{i}\right\rangle_{\mathrm{W}}+\left\langle\overline{\mathrm{u}}_{i}, \mathrm{p}_{\mathrm{f}_{k}}\right\rangle_{\mathrm{W}}\left\langle\dot{\overline{\mathrm{p}}}_{\mathrm{f}_{k}}, \mu_{i}\right\rangle_{\mathrm{W}}\right) \\
& +\sum_{i=1}^{\ell}\left(\left\langle\mathrm{u}_{i}, \dot{\overline{\mathrm{p}}}_{\mathrm{f}_{k}}\right\rangle_{\mathrm{W}}\left\langle\mathrm{p}_{\mathrm{f}_{k}}, \tilde{\mu}_{i}\right\rangle_{\mathrm{W}}+\left\langle\mathrm{u}_{i}, \overline{\mathrm{p}}_{\mathrm{f}_{k}}\right\rangle_{\mathrm{W}}\left\langle\dot{\mathrm{p}}_{\mathrm{f}_{k}}, \tilde{\mu}_{i}\right\rangle_{\mathrm{W}}\right) \quad \text { for } 1 \leq k \leq \mathrm{n} .
\end{aligned}
$$

From the first-order necessary optimality condition $\mathcal{L}_{\mathrm{f}_{k}}\left(\zeta^{*}\right)\left(f-\mathrm{f}_{k}^{*}\right) \geq 0$ for all $f \in \mathcal{F}$ and for $1 \leq k \leq \mathrm{n}$ we conclude that

$$
\begin{aligned}
& \sum_{i=1}^{\ell}\left(\left\langle\overline{\mathrm{u}}_{i}^{*}, \dot{\mathrm{p}}_{\mathrm{f}_{k}}^{*}\right\rangle_{\mathrm{W}}\left\langle\overline{\mathrm{p}}_{\mathrm{f}_{k}}^{*}, \mu_{i}^{*}\right\rangle_{\mathrm{W}}+\left\langle\overline{\mathrm{u}}_{i}^{*}, \mathrm{p}_{\mathrm{f}_{k}}^{*}\right\rangle_{\mathrm{W}}\left\langle\dot{\overline{\mathrm{f}}}_{\mathrm{f}_{k}}^{*}, \mu_{i}^{*}\right\rangle_{\mathrm{W}}\right) \\
& \quad+\sum_{i=1}^{\ell}\left(\left\langle\mathrm{u}_{i}^{*}, \dot{\overline{\mathrm{p}}}_{\mathrm{f}_{k}}^{*}\right\rangle_{\mathrm{W}}\left\langle\mathrm{p}_{\mathrm{f}_{k}}^{*}, \tilde{\mu}_{i}^{*}\right\rangle_{\mathrm{W}}+\left\langle\mathrm{u}_{i}^{*}, \overline{\mathrm{p}}_{\mathrm{f}_{k}}^{*}\right\rangle_{\mathrm{W}}\left\langle\dot{\mathrm{p}}_{\mathrm{f}_{k}}^{*}, \tilde{\mu}_{i}^{*}\right\rangle_{\mathrm{W}}\right)\left(f-\mathrm{f}_{k}\right) \geq 0
\end{aligned}
$$

for all $f \in \mathcal{F}$ and $1 \leq k \leq \mathrm{n}$.

Next we compute the derivative of the Lagrangian with respect to $\lambda_{i}$ for $1 \leq i \leq \ell$. Since $\lambda_{i} \in \mathbb{R}$ holds, we find

$$
\mathcal{L}_{\lambda_{i}}(\zeta)=-\left\langle\overline{\mathrm{u}}_{i}, \mu_{i}\right\rangle_{\mathrm{W}}-\left\langle\mathrm{u}_{i}, \tilde{\mu}_{i}\right\rangle_{\mathrm{W}} \quad \text { for } 1 \leq i \leq \ell .
$$

Utilizing $\mathcal{L}_{\lambda}\left(\zeta^{*}\right)=0$ we derive

$$
\left\langle\overline{\mathrm{u}}_{i}^{*}, \mu_{i}^{*}\right\rangle_{\mathrm{W}}+\left\langle\mathrm{u}_{i}^{*}, \tilde{\mu}_{i}^{*}\right\rangle_{\mathrm{W}}=0 \text { for } 1 \leq i \leq \ell .
$$

Now we investigate the derivative of the Lagrangian with respect to $\mathrm{u} \in\left(\mathbb{C}^{N}\right)^{\ell}$. First we note that

$$
\begin{aligned}
\mathrm{B}_{f, i j}^{\ell} & =\left\langle\mathcal{A}_{f} \psi_{j}, \bar{\psi}_{i}\right\rangle_{V^{\prime}, V}=\sum_{k=1}^{N} \sum_{l=1}^{N}\left(\overline{\mathrm{u}}_{i}\right)_{k}\left(\mathrm{u}_{j}\right)_{l}\left\langle\mathcal{A}_{f} \varphi_{l}, \bar{\varphi}_{k}\right\rangle_{V^{\prime}, V} \\
& =\sum_{k=1}^{N} \sum_{l=1}^{N}\left(\overline{\mathrm{u}}_{i}\right)_{k}\left(\mathrm{u}_{j}\right)_{l} \mathrm{~B}_{f, k l}=\overline{\mathrm{u}}_{i}^{\top} \mathrm{B}_{f} \mathrm{u}_{j}
\end{aligned}
$$

and

$$
\mathrm{s}_{f, i}^{\ell}=\left\langle\mathcal{S}_{f}, \bar{\psi}_{i}\right\rangle_{V^{\prime}, V}=\sum_{k=1}^{N} \overline{\mathrm{u}}_{i}\left\langle\mathcal{S}_{f}, \bar{\varphi}_{i}\right\rangle_{V^{\prime}, V}=\overline{\mathrm{u}}_{i}^{\top} \mathrm{s}_{f}
$$

for $1 \leq i, j \leq \ell$ and $f \in \mathcal{F}$. Thus, we find that

$$
\begin{aligned}
\left(\overline{\mathrm{B}_{f}^{\ell} \eta(f)-\mathrm{s}_{f}^{\ell}}\right)^{\top} \mathrm{q}(f) & =\sum_{i=1}^{\ell}\left(\sum_{j=1}^{\ell} \overline{\overline{\mathrm{u}}_{i}^{\top} \mathrm{B}_{f} \mathrm{u}_{j} \eta_{j}(f)}-\overline{\overline{\mathrm{u}}_{i}^{\top} \mathrm{s}_{f}}\right) \mathrm{q}_{i}(f) \\
& =\sum_{i=1}^{\ell}\left(\sum_{j=1}^{\ell}\left(\mathrm{u}_{i}^{\top} \overline{\mathrm{B}}_{f} \overline{\mathrm{u}}_{j} \bar{\eta}_{j}(f)\right)-\mathrm{u}_{i}^{\top} \overline{\mathrm{s}}_{f}\right) \mathrm{q}_{i}(f)
\end{aligned}
$$


and

$$
\left(\mathrm{B}_{f}^{\ell} \eta(f)-\mathrm{s}_{f}^{\ell}\right)^{\top} \tilde{\mathrm{q}}(f)=\sum_{i=1}^{\ell}\left(\sum_{j=1}^{\ell}\left(\overline{\mathrm{u}}_{i}^{\top} \mathrm{B}_{f} \mathrm{u}_{j} \eta_{j}(f)\right)-\overline{\mathrm{u}}_{i}^{\top} \mathrm{s}_{f}\right) \tilde{\mathrm{q}}_{i}(f)
$$

for $f \in \mathcal{F}$. Thus, we have

$$
\begin{aligned}
\mathcal{L}_{\mathrm{u}}(\zeta) \mathrm{u}_{\delta}= & -\int_{\mathcal{F}} \frac{\beta}{2} \sum_{i=1}^{\ell} \eta_{i}(f)\left\langle\mathrm{u}_{\delta, i}, \overline{\mathrm{p}}(f)-\sum_{j=1}^{\ell} \bar{\eta}_{j}(f) \overline{\mathrm{u}}_{j}\right\rangle_{\mathrm{W}} \mathrm{d} f \\
& +\int_{\mathcal{F}} \sum_{i=1}^{\ell}\left(\sum_{j=1}^{\ell}\left(\mathrm{u}_{\delta, i}^{\top} \overline{\mathrm{B}}_{f} \overline{\mathrm{u}}_{j} \bar{\eta}_{j}(f)\right)-\mathrm{u}_{\delta, i}^{\top} \overline{\mathrm{s}}_{f}\right) \mathrm{q}_{i}(f) \mathrm{d} f \\
& +\int_{\mathcal{F}} \sum_{i=1}^{\ell} \sum_{j=1}^{\ell} \overline{\mathrm{u}}_{i}^{\top} \mathrm{B}_{f} \mathrm{u}_{\delta, j} \eta_{j}(f) \tilde{\mathrm{q}}_{i}(f) \mathrm{d} f \\
& +\sum_{i=1}^{\ell}\left(\left\langle\left(\mathcal{R}(\mathrm{f})-\lambda_{i}\right) \mathrm{u}_{\delta, i}, \tilde{\mu}_{i}\right\rangle_{\mathrm{W}}-\left\langle\mathrm{u}_{\delta, i}, \overline{\mathrm{u}}_{i}\right\rangle_{\mathrm{W}} \phi_{i}\right)
\end{aligned}
$$

for an arbitrary direction $\mathrm{u}_{\delta}=\left(\mathrm{u}_{\delta, 1}, \ldots, \mathrm{u}_{\delta, \ell}\right) \in\left(\mathbb{C}^{N}\right)^{\ell}$. For $f \in \mathcal{F}$ we set $\mathrm{p}^{\ell}(f)=$ $\sum_{j=1}^{\ell} \eta_{j}(f) \mathrm{u}_{j} \in \mathbb{C}^{N}$ and $\tilde{\mathrm{q}}^{\ell}(f)=\sum_{i=1}^{\ell} \tilde{\mathrm{q}}_{i}(f) \overline{\mathrm{u}}_{i} \in \mathbb{C}^{N}$. Then, we have

$$
\sum_{i=1}^{\ell}\left(\sum_{j=1}^{\ell}\left(\mathrm{u}_{\delta, i}^{\top} \overline{\mathrm{B}}_{f} \overline{\mathrm{u}}_{j} \bar{\eta}_{j}(f)\right)-\mathrm{u}_{\delta, i}^{\top} \overline{\mathrm{s}}_{f}\right) \mathrm{q}_{i}(f)=\sum_{i=1}^{\ell} \mathrm{q}_{i}(f) \mathrm{u}_{\delta, i}^{\top} \overline{\left(\mathrm{B}_{f} \mathrm{p}^{\ell}(f)-\mathrm{s}_{f}\right)}
$$

and

$$
\sum_{i=1}^{\ell} \overline{\mathrm{u}}_{i}^{\top} \mathrm{B}_{f} \mathrm{u}_{\delta, j} \eta_{j}(f) \tilde{\mathrm{q}}_{i}(f)=\eta_{j}(f) \tilde{\mathrm{q}}^{\ell}(f)^{\top} \mathrm{B}_{f} \mathrm{u}_{\delta, j}=\eta_{j}(f) \mathrm{u}_{\delta, j}^{\top} \mathrm{B}_{f}^{\top} \tilde{\mathrm{q}}^{\ell}(f) .
$$

Hence, we derive

$$
\begin{aligned}
\mathcal{L}_{\mathrm{u}}(\zeta) \mathrm{u}_{\delta}= & \sum_{i=1}^{\ell} \int_{\mathcal{F}} \mathrm{q}_{i}(f) \mathrm{u}_{\delta, i}^{\top} \overline{\left(\mathrm{B}_{f} \mathrm{p}^{\ell}(f)-\mathrm{s}_{f}\right)} \mathrm{d} f \\
& +\sum_{i=1}^{\ell} \int_{\mathcal{F}} \eta_{i}(f) \mathrm{u}_{\delta, i}^{\top}\left(\mathrm{B}_{f}^{\top} \tilde{\mathrm{q}}^{\ell}(f)-\frac{\beta}{2} \mathrm{~W}\left(\overline{\mathrm{p}}(f)-\overline{\mathrm{p}}^{\ell}(f)\right)\right) \mathrm{d} f \\
& +\sum_{i=1}^{\ell}\left(\left\langle\left(\mathcal{R}(\mathrm{f})-\lambda_{i}\right) \mathrm{u}_{\delta, i}, \tilde{\mu}_{i}\right\rangle_{\mathrm{W}}-\left\langle\mathrm{u}_{\delta, i}, \phi_{i} \overline{\mathrm{u}}_{i}\right\rangle_{\mathrm{W}}\right)
\end{aligned}
$$

for all $\mathrm{u}_{\delta} \in\left(\mathbb{C}^{N}\right)^{\ell}$. Utilizing the first-order necessary optimality condition $\mathcal{L}_{\mathrm{u}}\left(\zeta^{*}\right)=$ $0, \phi_{i}^{*} \in \mathbb{R}$ for $1 \leq i \leq \ell, \mathrm{B}_{f}^{\mathrm{H}}=\overline{\mathrm{B}}_{f}^{\top}$ and $\mathrm{A} .3$ we obtain

$$
\begin{aligned}
0= & \sum_{i=1}^{\ell} \mathrm{u}_{\delta, i}^{\top} \int_{\mathcal{F}} \mathrm{q}_{i}^{*}(f) \overline{\left(\mathrm{B}_{f} \mathrm{p}^{\ell, *}(f)-\mathrm{s}_{f}\right)} \mathrm{d} f \\
& +\sum_{i=1}^{\ell} \mathrm{u}_{\delta, i}^{\top} \int_{\mathcal{F}} \eta_{i}^{*}(f) \overline{\left(\mathrm{B}_{f}^{\mathrm{H}} \mathrm{q}^{\ell, *}(f)-\frac{\beta}{2} \mathrm{~W}\left(\mathrm{p}(f)-\mathrm{p}^{\ell, *}(f)\right)\right)} \mathrm{d} f \\
& +\sum_{i=1}^{\ell}\left(\left\langle\left(\mathcal{R}\left(\mathrm{f}^{*}\right)-\lambda_{i}^{*}\right) \mathrm{u}_{\delta, i}, \tilde{\mu}_{i}^{*}\right\rangle_{\mathrm{W}}-\left\langle\mathrm{u}_{\delta, i}, \phi_{i}^{*} \overline{\mathrm{u}}_{i}^{*}\right\rangle_{\mathrm{W}}\right)
\end{aligned}
$$


for all $\mathrm{u}_{\delta} \in\left(\mathbb{C}^{N}\right)^{\ell}$, where we have set $\mathrm{q}^{\ell, *}(f)=\sum_{i=1}^{\ell} \mathrm{q}_{i}^{*}(f) \mathrm{u}_{i}^{*}$. Analogous to 3.1 we deduce that

$$
\langle\mathcal{R}(\mathrm{f}) \mathrm{u}, \overline{\mathrm{v}}\rangle_{\mathrm{W}}=\langle\mathrm{u}, \overline{\mathcal{R}(\mathrm{f}) \mathrm{v}}\rangle_{\mathrm{W}} \quad \text { for all } \mathrm{u}, \mathrm{v} \in \mathbb{C}^{N} .
$$

We define the vectors $\mathrm{g}_{i}^{*} \in \mathbb{C}^{N}, 1 \leq i \leq \ell$, by

$$
\begin{aligned}
\mathrm{g}_{i}^{*}= & \int_{\mathcal{F}} \mathrm{q}_{i}^{*}(f) \overline{\left(\mathrm{B}_{f} \mathrm{p}^{\ell, *}(f)-\mathrm{s}_{f}\right)} \mathrm{d} f \\
& +\int_{\mathcal{F}} \eta_{i}^{*}(f) \overline{\left(\mathrm{B}_{f}^{\mathrm{H}} \mathrm{q}^{\ell, *}(f)-\frac{\beta}{2} \mathrm{~W}\left(\mathrm{p}(f)-\mathrm{p}^{\ell, *}(f)\right)\right.} \mathrm{d} f
\end{aligned}
$$

and set $\mathrm{G}_{i}^{*}=\mathrm{W}^{-1} \overline{\mathrm{g}}_{i}^{*} \in \mathbb{C}^{N}$ for $1 \leq i \leq \ell$. Using $\mathrm{g}_{i}^{*}=\mathrm{W}_{\overline{\mathrm{G}}}^{*}$ we deduce

$$
\mathrm{u}_{\delta, i}^{\top} \mathrm{g}_{i}^{*}=\mathrm{u}_{\delta, i}^{\top} \mathrm{W} \overline{\mathrm{G}}_{i}^{*}=\left\langle\mathrm{u}_{\delta, i}, \overline{\mathrm{G}}_{i}^{*}\right\rangle_{\mathrm{W}} .
$$

Thus, $\phi_{i}^{*} \in \mathbb{R}$ and A.6 imply for all $i \in\{1, \ldots, \ell\}$ that

$$
\begin{aligned}
& 0=\left\langle\mathrm{u}_{\delta, i}, \overline{\mathrm{G}}_{i}^{*}\right\rangle_{\mathrm{W}}+\left\langle\left(\mathcal{R}\left(\mathrm{f}^{*}\right)-\lambda_{i}^{*}\right) \mathrm{u}_{\delta, i}, \tilde{\mu}_{i}^{*}\right\rangle_{\mathrm{W}}-\left\langle\mathrm{u}_{\delta, i}, \phi_{i}^{*} \overline{\mathrm{u}}_{i}^{*}\right\rangle_{\mathrm{W}} \\
& \left.=\left\langle\mathrm{u}_{\delta, i}, \overline{\mathrm{G}}_{i}^{*}\right\rangle_{\mathrm{W}}+\left\langle\mathrm{u}_{\delta, i}, \overline{\left(\mathcal{R}(\mathrm{f} *)-\lambda_{i}^{*}\right) \overline{\tilde{\mu}}_{i}^{*}}\right\rangle_{\mathrm{W}}-\left\langle\mathrm{u}_{\delta, i}, \phi_{i}^{*} \overline{\mathrm{u}}_{i}^{*}\right\rangle_{\mathrm{W}}\right) \\
& \left.=\left\langle\mathrm{u}_{\delta, i}, \overline{\mathrm{G}_{i}^{*}+\left(\mathcal{R}(\mathrm{f} *)-\lambda_{i}^{*}\right) \overline{\tilde{\mu}}_{i}^{*}-\phi_{i}^{*} \mathrm{u}_{i}^{*}}\right\rangle_{\mathrm{W}}\right)
\end{aligned}
$$

for all $\mathrm{u}_{\delta, i} \in \mathbb{C}^{N}$. Thus, we obtain

$$
\left(\mathcal{R}\left(\mathrm{f}^{*}\right)-\lambda_{i}^{*}\right) \overline{\tilde{\mu}}_{i}^{*}=-\mathrm{G}_{i}^{*}+\phi_{i}^{*} \mathrm{u}_{i}^{*} \quad \text { in } \mathbb{C}^{N} \text { for } 1 \leq i \leq \ell .
$$

Combining A.6, A.8 and using $\mathcal{R}\left(\mathrm{f}^{*}\right) \mathrm{u}_{i}^{*}=\lambda_{i}^{*} \mathrm{u}_{i}^{*}$ we find that

$$
\begin{aligned}
& \phi_{i}^{*}=\phi_{i}^{*}\left|\mathrm{u}_{i}^{*}\right|_{\mathrm{W}}^{2}=\left\langle\phi_{i}^{*} \mathrm{u}_{i}^{*}, \overline{\mathrm{u}}_{i}^{*}\right\rangle_{\mathrm{W}}=\left\langle\left(\mathcal{R}\left(\mathrm{f}^{*}\right)-\lambda_{i}^{*}\right) \overline{\tilde{\mu}}_{i}^{*}+\mathrm{G}_{i}^{*}, \overline{\mathrm{u}}_{i}^{*}\right\rangle_{\mathrm{W}} \\
& =\left\langle\left(\mathcal{R}\left(\mathrm{f}^{*}\right)-\lambda_{i}^{*}\right) \overline{\tilde{\mu}}_{i}^{*}, \overline{\mathrm{u}}_{i}^{*}\right\rangle_{\mathrm{W}}+\left\langle\mathrm{G}_{i}^{*}, \overline{\mathrm{u}}_{i}^{*}\right\rangle_{\mathrm{W}} \\
& =\left\langle\overline{\tilde{\mu}}_{i}^{*}, \overline{\left(\mathcal{R}\left(\mathrm{f}^{*}\right)-\lambda_{i}^{*}\right) \mathrm{u}_{i}^{*}}\right\rangle_{\mathrm{W}}+\left\langle\mathrm{G}_{i}^{*}, \overline{\mathrm{u}}_{i}^{*}\right\rangle_{\mathrm{W}}=\left\langle\mathrm{G}_{i}^{*}, \overline{\mathrm{u}}_{i}^{*}\right\rangle_{\mathrm{W}}
\end{aligned}
$$

for $1 \leq i \leq \ell$. Hence, A.8 can be expressed as

$$
\left(\mathcal{R}\left(\mathrm{f}^{*}\right)-\lambda_{i}^{*}\right) \overline{\tilde{\mu}}_{i}^{*}=-\left(\mathrm{G}_{i}^{*}-\left\langle\mathrm{G}_{i}^{*}, \overline{\mathrm{u}}_{i}^{*}\right\rangle_{\mathrm{W}} \mathrm{u}_{i}^{*}\right) \in \operatorname{span}\left\{\mathrm{u}_{i}^{*}\right\}^{\perp} \text { for } 1 \leq i \leq \ell .
$$

Finally, we compute the derivative of the Lagrangian with respect to $\overline{\mathrm{u}}$. We obtain

$$
\begin{aligned}
\mathcal{L}_{\overline{\mathrm{u}}}(\zeta) \tilde{\mathrm{u}}_{\delta}= & -\int_{\mathcal{F}} \frac{\beta}{2} \sum_{i=1}^{\ell} \bar{\eta}_{i}(f)\left\langle\mathrm{p}(f)-\sum_{j=1}^{\ell} \eta_{j}(f) \mathrm{u}_{i}, \tilde{\mathrm{u}}_{\delta, i}\right\rangle_{\mathrm{W}} \mathrm{d} f \\
& +\int_{\mathcal{F}} \sum_{i=1}^{\ell} \sum_{j=1}^{\ell} \mathrm{u}_{i}^{\top} \overline{\mathrm{B}}_{f} \tilde{\mathrm{u}}_{\delta, j} \bar{\eta}_{j}(f) \mathrm{q}_{i}(f) \mathrm{d} f \\
& +\int_{\mathcal{F}} \sum_{i=1}^{\ell}\left(\sum_{j=1}^{\ell} \tilde{\mathrm{u}}_{\delta, i}^{\top} \mathrm{B}_{f} \mathrm{u}_{j} \eta_{j}(f)-\tilde{\mathrm{u}}_{\delta, i}^{\top} \mathrm{s}_{f}\right) \tilde{\mathrm{q}}_{i}(f) \mathrm{d} f \\
& \left.+\sum_{i=1}^{\ell}\left(\overline{\left\langle\left(\mathcal{R}(\mathrm{f})-\lambda_{i}\right)\right.} \tilde{\mathrm{u}}_{\delta, i}, \mu_{i}\right\rangle_{\mathrm{W}}-\left\langle\mathrm{u}_{i}, \tilde{\mathrm{u}}_{\delta, i}\right\rangle_{\mathrm{W}} \phi_{i}\right)
\end{aligned}
$$


for any direction $\tilde{\mathrm{u}}_{\delta}=\left(\tilde{\mathrm{u}}_{\delta, 1}, \ldots, \tilde{\mathrm{u}}_{\delta, \ell}\right) \in\left(\mathbb{C}^{N}\right)^{\ell}$. Again we set $\mathrm{p}^{\ell}(f)=\sum_{j=1}^{\ell} \eta_{j}(f) \mathrm{u}_{j}$ and $\mathrm{q}^{\ell}(f)=\sum_{i=1}^{\ell} \mathrm{q}_{i}(f) \mathrm{u}_{i}$. Then, it follows that

$$
\begin{aligned}
\sum_{i=1}^{\ell} \mathrm{u}_{i}^{\top} \overline{\mathrm{B}}_{f} \tilde{\mathrm{u}}_{\delta, j} \bar{\eta}_{j}(f) \mathrm{q}_{i}(f) & =\left(\sum_{i=1}^{\ell} \mathrm{q}_{i}(f) \mathrm{u}_{i}\right)^{\top} \overline{\mathrm{B}}_{f} \tilde{\mathrm{u}}_{\delta, j} \bar{\eta}_{j}(f)=\mathrm{q}^{\ell}(f)^{\top} \overline{\mathrm{B}}_{f} \tilde{\mathrm{u}}_{\delta, j} \bar{\eta}_{j}(f) \\
& =\bar{\eta}_{j}(f)\left(\overline{\mathrm{B}}_{f}^{\top} \mathrm{q}^{\ell}(f)\right)^{\top} \tilde{\mathrm{u}}_{\delta, j}=\bar{\eta}_{j}(f)\left(\mathrm{B}_{f}^{\mathrm{H}} \mathrm{q}^{\ell}(f)\right)^{\top} \tilde{\mathrm{u}}_{\delta, j} \\
\sum_{j=1}^{\ell} \tilde{\mathrm{u}}_{\delta, i}^{\top} \mathrm{B}_{f} \mathrm{u}_{j} \eta_{j}(f) \tilde{\mathrm{q}}_{i}(f) & =\tilde{\mathrm{u}}_{\delta, i}^{\top} \mathrm{B}_{f}\left(\sum_{j=1}^{\ell} \eta_{j}(f) \mathrm{u}_{j}\right) \tilde{\mathrm{q}}_{i}(f)=\tilde{\mathrm{u}}_{\delta, i}^{\top} \mathrm{B}_{f} \mathrm{p}^{\ell}(f) \tilde{\mathrm{q}}_{i}(f) \\
& =\left(\mathrm{B}_{f} \mathrm{p}^{\ell}(f)\right)^{\top} \tilde{\mathrm{u}}_{\delta, i} \tilde{\mathrm{q}}_{i}(f)
\end{aligned}
$$

for $f \in \mathcal{F}$. Recall that $\overline{\mathrm{W}}^{\top}=\mathrm{W}$ holds. Thus, we find

$$
\begin{aligned}
\mathcal{L}_{\overline{\mathrm{u}}}(\zeta) \tilde{\mathrm{u}}_{\delta}= & \sum_{i=1}^{\ell} \tilde{\mathrm{u}}_{\delta, i}^{\top} \int_{\mathcal{F}} \bar{\eta}_{i}(f)\left(\mathrm{B}_{f}^{\mathrm{H}} \mathrm{q}^{\ell}(f)-\frac{\beta}{2} \mathrm{~W}\left(\mathrm{p}(f)-\mathrm{p}^{\ell}(f)\right)\right) \mathrm{d} f \\
& +\sum_{i=1}^{\ell} \tilde{\mathrm{u}}_{\delta, i}^{\top} \int_{\mathcal{F}} \tilde{\mathrm{q}}_{i}(f)\left(\left(\mathrm{B}_{f} \mathrm{p}^{\ell}(f)-\mathrm{s}_{f}\right)\right) \mathrm{d} f \\
& \left.+\sum_{i=1}^{\ell}\left(\overline{\left\langle\left(\mathcal{R}(\mathrm{f})-\lambda_{i}\right)\right.} \tilde{\mathrm{u}}_{\delta, i}, \mu_{i}\right\rangle_{\mathrm{W}}-\left\langle\mathrm{u}_{i}, \tilde{\mathrm{u}}_{\delta, i}\right\rangle_{\mathrm{W}} \phi_{i}\right)
\end{aligned}
$$

for any direction $\tilde{\mathrm{u}}_{\delta} \in\left(\mathbb{C}^{N}\right)^{\ell}$. Using A.3, A.6 and A.7 the first-order necessary optimality condition $\mathcal{L}_{\overline{\mathrm{u}}}\left(\zeta^{*}\right)=0$ implies that

$$
\begin{aligned}
0 & =\sum_{i=1}^{\ell}\left(\tilde{\mathrm{u}}_{\delta, i}^{\top} \overline{\mathrm{g}}_{i}^{*}+\overline{\left\langle\left(\mathcal{R}\left(\mathrm{f}^{*}\right)-\lambda_{i}^{*}\right) \overline{\tilde{\mathrm{u}}}_{\delta, i}, \bar{\mu}_{i}^{*}\right\rangle_{\mathrm{W}}}-\left\langle\tilde{\mathrm{u}}_{\delta, i}, \phi_{i}^{*} \mathrm{u}_{i}^{*}\right\rangle_{\mathrm{W}}\right) \\
& =\sum_{i=1}^{\ell}\left(\tilde{\mathrm{u}}_{\delta, i}^{\top} \mathrm{WG}_{i}^{*}+\left\langle\tilde{\mathrm{u}}_{\delta, i},\left(\mathcal{R}\left(\mathrm{f}^{*}\right)-\lambda_{i}^{*}\right) \mu_{i}^{*}-\phi_{i}^{*} \mathrm{u}_{i}^{*}\right\rangle_{\mathrm{W}}\right) \\
& =\sum_{i=1}^{\ell}\left\langle\tilde{\mathrm{u}}_{\delta, i},\left(\mathcal{R}\left(\mathrm{f}^{*}\right)-\lambda_{i}^{*}\right) \mu_{i}^{*}+\mathrm{G}_{i}^{*}-\phi_{i}^{*} \mathrm{u}_{i}^{*}\right\rangle_{\mathrm{W}}
\end{aligned}
$$

for any direction $\tilde{\mathrm{u}}_{\delta} \in\left(\mathbb{C}^{N}\right)^{\ell}$, where we have used $\overline{\mathrm{g}}_{i}^{*}=\mathrm{WG}_{i}^{*}$. From A.9 we conclude

$$
\left(\mathcal{R}\left(\mathrm{f}^{*}\right)-\lambda_{i}^{*}\right) \mu_{i}^{*}=-\left(\mathrm{G}_{i}^{*}+\left\langle\mathrm{G}_{i}^{*}, \overline{\mathrm{u}}_{i}^{*}\right\rangle_{\mathrm{W}} \mathrm{u}_{i}^{*}\right) \in \operatorname{span}\left\{\mathrm{u}_{i}^{*}\right\}^{\perp} \quad \text { for } 1 \leq i \leq \ell .
$$

From A.10 and A.11 we obtain that

$$
\left(\mathcal{R}\left(\mathrm{f}^{*}\right)-\lambda_{i}^{*}\right) \mu_{i}^{*}=\left(\mathcal{R}\left(\mathrm{f}^{*}\right)-\lambda_{i}^{*}\right) \overline{\tilde{\mu}}_{i}^{*} \in \operatorname{span}\left\{\mathrm{u}_{i}^{*}\right\}^{\perp} .
$$

Since the linear equation $\left(\mathcal{R}\left(\mathrm{f}^{*}\right)-\lambda_{i}^{*}\right) \mu_{i}=\mathrm{r}_{i}$ is uniquely solvable for every righthand side $\mathrm{r}_{i} \in \operatorname{span}\left\{\mathrm{u}_{i}^{*}\right\}^{\perp}$ we conclude that

$$
\tilde{\mu}_{i}^{*}=\bar{\mu}_{i}^{*} \quad \text { for } 1 \leq i \leq \ell .
$$

From A.5 and A.12 we infer that

$$
0=\left\langle\overline{\mathrm{u}}_{i}^{*}, \mu_{i}^{*}\right\rangle_{\mathrm{W}}+\left\langle\mathrm{u}_{i}^{*}, \bar{\mu}_{i}^{*}\right\rangle_{\mathrm{W}}=\left\langle\overline{\mathrm{u}}_{i}^{*}, \mu_{i}^{*}\right\rangle_{\mathrm{W}}+{\overline{\left\langle\overline{\mathrm{u}}_{i}^{*}, \mu_{i}^{*}\right\rangle}}_{\mathrm{W}}=2 \Re e\left(\left\langle\overline{\mathrm{u}}_{i}^{*}, \mu_{i}^{*}\right\rangle_{\mathrm{W}}\right)
$$

for $1 \leq i \leq \ell$, which implies

$$
\Re e\left(\left\langle\overline{\mathrm{u}}_{i}^{*}, \mu_{i}^{*}\right\rangle_{\mathrm{W}}\right)=\Re e\left(\left\langle\mathrm{u}_{i}^{*}, \bar{\mu}_{i}^{*}\right\rangle_{\mathrm{W}}\right)=0 \quad \text { for } 1 \leq i \leq \ell .
$$


see 4.4c. Furthermore, A.12 implies that

$$
\begin{aligned}
& \mathcal{L}_{\mathrm{f}_{k}}\left(\zeta^{*}\right)=\sum_{i=1}^{\ell}\left(\left\langle\overline{\mathrm{u}}_{i}^{*}, \dot{\mathrm{p}}_{k}^{*}\right\rangle_{\mathrm{W}}\left\langle\overline{\mathrm{p}}_{k}^{*}, \mu_{i}^{*}\right\rangle_{\mathrm{W}}+\left\langle\overline{\mathrm{u}}_{i}^{*}, \mathrm{p}_{k}^{*}\right\rangle_{\mathrm{W}}\left\langle\dot{\mathrm{p}}_{k}^{*}, \mu_{i}^{*}\right\rangle_{\mathrm{W}}\right) \\
& +\sum_{i=1}^{\ell}\left(\left\langle\mathrm{u}_{i}^{*}, \dot{\overline{\mathrm{p}}}_{k}^{*}\right\rangle_{\mathrm{W}}\left\langle\mathrm{p}_{k}^{*}, \bar{\mu}_{i}^{*}\right\rangle_{\mathrm{W}}+\left\langle\mathrm{u}_{i}^{*}, \overline{\mathrm{p}}_{k}^{*}\right\rangle_{\mathrm{W}}\left\langle\dot{\mathrm{p}}_{k}^{*}, \bar{\mu}_{i}^{*}\right\rangle_{\mathrm{W}}\right) \\
& =2 \Re e\left(\sum_{i=1}^{\ell}\left(\left\langle\overline{\mathrm{u}}_{i}^{*}, \dot{\mathrm{p}}_{k}^{*}\right\rangle_{\mathrm{W}}\left\langle\overline{\mathrm{p}}_{k}^{*}, \mu_{i}^{*}\right\rangle_{\mathrm{W}}+\left\langle\overline{\mathrm{u}}_{i}^{*}, \mathrm{p}_{k}^{*}\right\rangle_{\mathrm{W}}\left\langle\dot{\overline{\mathrm{p}}}_{k}^{*}, \mu_{i}^{*}\right\rangle_{\mathrm{W}}\right)\right) \\
& =2 \Re e\left(\sum_{i=1}^{\ell}\left(\left\langle\mathrm{u}_{i}^{*}, \dot{\overline{\mathrm{p}}}_{k}^{*}\right\rangle_{\mathrm{W}}\left\langle\mathrm{p}_{k}^{*}, \bar{\mu}_{i}^{*}\right\rangle_{\mathrm{W}}+\left\langle\mathrm{u}_{i}^{*}, \overline{\mathrm{p}}_{k}^{*}\right\rangle_{\mathrm{W}}\left\langle\dot{\mathrm{p}}_{k}^{*}, \bar{\mu}_{i}^{*}\right\rangle_{\mathrm{W}}\right)\right) \quad \text { for } 1 \leq k \leq \mathrm{n},
\end{aligned}
$$

which implies $4.4 \mathrm{~d}$.

Remark A.1. Let us mention that the first-order necessary optimality conditions can also be derived by considering the following Lagrange functional $\tilde{\mathcal{L}}: x \times y \rightarrow \mathbb{R}$ defined as:

$$
\begin{aligned}
& \tilde{\mathcal{L}}(\theta)=\frac{\beta}{2} \int_{\mathcal{F}}\left\langle\mathrm{p}(f)-\sum_{i=1}^{\ell} \eta_{i}(f) \mathrm{u}_{i}, \overline{\mathrm{p}}(f)-\sum_{j=1}^{\ell} \bar{\eta}_{j}(f) \overline{\mathrm{u}}_{j}\right\rangle_{\mathrm{W}} \mathrm{d} f \\
& +2 \Re e\left(\int_{\mathcal{F}}\left(\overline{\mathrm{B}_{f}^{\ell} \eta(f)-\mathrm{s}_{f}^{\ell}}\right)^{\top} \mathrm{q}(f) \mathrm{d} f+\sum_{i=1}^{\ell}\left\langle\left(\overline{\left.\mathcal{R}(\mathrm{f})-\lambda_{i}\right)} \overline{\mathrm{u}}_{i}, \mu_{i}\right\rangle_{\mathrm{W}}\right)+\sum_{i=1}^{\ell}\left(1-\left|\mathrm{u}_{i}\right|_{\mathrm{W}}^{2}\right) \phi_{i}\right.
\end{aligned}
$$

for $\theta=(z, \xi), z=(\eta, \mathrm{f}, \mathrm{u}, \lambda) \in \mathcal{X}$ and $\xi=(\mathrm{q}, \mu, \phi) \in \mathcal{y}$.

\section{REFERENCES}

[1] A. Alla and M. Falcone. An adaptive POD approximation method for the control of advectiondiffusion equations, submitted (2012).

[2] P. Bouboulis. Wirtinger's Calculus in general Hilbert Spaces, CoRR, abs/1005.5170 (2010).

[3] T. Bui-Thanh. "Model-constrained optimization methods for reduction of parameterized systems", Ph.D. thesis, MIT, USA (2007).

[4] T. Bui-Thanh, M. Damodoran and K. Willcox. Aerodynamic data reconstruction and inverse design using proper orthogonal decomposition, American Institute of Aeronautics and Astronautics (AIAA) Journal, 42 (2004), 1505-1516.

[5] L.C. Evans. "Partial Differential Equations", American Mathematical Society, Providence, Rhode Island (2002).

[6] R. Everson and L. Sirovich. The Karhunen-Loève precedure for gappy data, Journal of the Optical Society of America, 12 (1995), 1657-1664.

[7] M.A. Grepl, Y. Maday, N.C. Nguyen and A.T. Patera. Efficient reduced-basis treatment of affine and nonlinear partial differential equations, ESAIM: Mathematical Modelling and Numerical Analysis, 41 (2007), 575-605.

[8] K. Fukunaga. "Introduction to Statistical Recognition", Academic Press, New York, USA, 1990.

[9] A. Hepberger, F. Diwoky, H.-H. Priebsch and S. Volkwein. Impedance identification out of pressure datas with a hybrid measurement-simulation methodology up to $1 \mathrm{kHz}$, In: Proceedings of the International Conference on Noise and Engineering, Leuven, Belgium (2006).

[10] P. Holmes, J.L. Lumley, and G. Berkooz. "Turbulence, Coherent Structures, Dynamical Systems and Symmetry", Cambridge Monographs on Mechanics, Cambridge University Press, 1996.

[11] K. Ito and S.S. Ravindran. A reduced-order method for simulation and control of fluid flows, Journal of Computational Physics, 143 (1998), 403-425. 
[12] M. Kahlbacher and S. Volkwein. Galerkin proper orthogonal decomposition methods for parameter dependent elliptic systems, Discussiones Mathematicae: Differential Inclusions, Control and Optimization, 27 (2007), 95-117.

[13] K. Kunisch and S. Volkwein. Control of Burgers' equation by areduced order approach using proper orthogonal decomposition, Journal of Optimization Theory and Applications, 102 (1999), 345-371.

[14] K. Kunisch and S. Volkwein. Galerkin proper orthogonal decomposition methods for a general equation in fluid dynamics, SIAM Journal on Numerical Analysis, 40 (2002), 492-515.

[15] K. Kunisch and S. Volkwein. Optimal snapshot location for computing POD basis functions, ESAIM: Mathematical Modelling and Numerical Analysis, 44 (2010), 509-529.

[16] H.V. Ly and H.T. Tran. Proper orthogonal decomposition for flow calculations and optimal control in a horizontal CVD reactor, Quarterly of Applied Mathematics, 60 (2002), 631-656.

[17] H. Maurer and J. Zowe. First and second-order necessary and sufficient optimality conditions for infinite-dimensional programming problems. Mathematical Programming, 16 (1979), 98110.

[18] J. Nocedal and S. Wright. "Numerical Optimization", Springer Series in Operation Research, second edition, Springer, New York (2006).

[19] A.T. Patera and G. Rozza. "Reduced Basis Approximation and A-Posteriori Error Estimation for Parametrized Partial Differential Equations", MIT-Pappalardo Graduate Monographs in Mechanical Engineering, 2006.

[20] S.S. Ravindran. Adaptice reduced-order controllers for a thermal flow system using proper orthogonal decomposition, International Journal of Bifurcation and Chaos, 15 (2002), 9971013.

[21] R. Remmert. "Theory of Complex Functions", fourth corrected printing, Springer, New York Berlin Heidelberg, 1998.

[22] S. Volkwein. Admittance identification from point-wise sound pressure measurements using reduced-order modelling, Journal of Optimization Theory and Applications, 14 (2010), 166193.

[23] S. Volkwein. Model Reduction using Proper Orthogonal Decomposition, Lecture Notes, University of Constance (2011).

[24] S. Volkwein and A. Hepberger. Impedance identification by POD model reduction techniques, at-Automatisierungstechnik, 8 (2008), 437-446.

Received xxxx 20xx; revised xxxx 20xx.

E-mail address: Oliver.Lass@uni-konstanz.de

E-mail address: Stefan.Volkwein@uni-konstanz.de 